\title{
Resonance Theory in Atom-Surface Scattering
}

\section{Gerard}

Centre de Mathematiques, Unité de Recherche Associée D 0169, Ecole Polytechnique F-91128 Palaiseau Cedex, France

\begin{abstract}
We study the problem of analytic extension of the resolvent for Hamiltonians arising in scattering of atoms by a quantum surface. We prove that the resolvent extends holomorphically to some regions of the lower half plane with isolated singularities called Landau resonances which are branch points of the resolvent. We study also the effect of impurities on the singularities of the resolvent and show that the presence of impurities adds poles to the Landau resonances.
\end{abstract}

\section{Introduction}

We study in this paper the theory of resonances for Hamiltonians arising in atom-surface scattering. The theory of atomic or molecular collisions with surfaces has been greatly developed by chemists in recent years since the measurement of scattering of atoms by a quantum surface is a way to study the surface structure of materials at atomic scales (see [Ge] for a review).

The typical form of the Hamiltonian is the following: $H=-\Delta+V(x, y)$ on $\mathbf{R}_{x}^{m-1} \times \mathbf{R}_{y}$, where $y$ is the direction normal to the surface and $V(x, y)$ is an effective potential describing the interaction between an atom and a crystalline or non-crystalline material. Strictly speaking this Hamiltonian corresponds to a thin slab of material since it is possible for atoms to pass through the crystal. The Hamiltonian corresponding to an impenetrable material is the following: $H^{\prime}=-\Delta+V(x, y)$ on $\mathbf{R}_{x}^{m-1} \times \mathbf{R}_{y}^{+}$with Dirichlet boundary condition on $y=0$.

We will concentrate on $H$ but all results proved for $H$ hold for $H^{\prime}$ under corresponding hypotheses. (The proofs can be adapted almost verbatim.) When the surface is a perfect crystal, $V(x, y)$ tends to zero when $y$ tends to infinity and is periodic in $x$ with respect to some lattice $T$ in $\mathbf{R}^{m-1}$. In this paper we will always assume that $V$ is exponentially decreasing in $y$ in a suitable sense.

For crystalline surfaces, $H$ is usually studied using Bloch's theory to reduce the study of the resolvent $(H-\lambda)^{-1}$ to the study of $\left(H_{p}-\lambda\right)^{-1}$, where the Bloch number $p$ belongs to a fundamental domain of the dual lattice $T^{*}$ and $H_{p}=D_{y}^{2}+\left(D_{x}+p\right)^{2}+V(x, y), \quad D_{x_{i}}=(1 / i) \partial / \partial x_{i}, \quad D_{y}=(1 / i) \partial / \partial y$ is a reduced 
Hamiltonian on a cylinder $C_{T}=F_{T} \times \mathbf{R}$, where $F_{T}$ is a fundamental domain of $T$.

It turns out that under classical hypotheses like exponential decay in $y$ of $V(x, y)$ or dilation analyticity in $y$, it is possible to extend meromorphically $\left(H_{p}-\lambda\right)^{-1}$ to some region in the lower half plane with poles at the resonances of $H_{p}$. This kind of problem has been studied numerically. (See for example [Mo].)

Since $H$ is obtained as a direct integral of $H_{p}$ over $p$, one is naturally led to the guess that all resonances of $H_{p}$ are singularities of $(H-\lambda)^{-1}$, which in the physical case $m=3$ would lead to some closed set of positive measure as singularities of $(H-\lambda)^{-1}$.

The main result of this paper is that this guess is wrong.

We will consider two kinds of problems:

i) Local Extension Problem: given $\lambda_{0} \in \sigma(H)$, extend analytically $(H-\lambda)^{-1}$ to a small neighborhood of $\lambda_{0}$ and describe its singularities.

ii) Global Extension Problem: extend analytically $(H-\lambda)^{-1}$ to some given open set $\mathscr{U}$ in $\mathbf{C}$ and describe its singularities.

For the local extension problem, we prove that for any $\lambda_{0} \in \sigma(H)$, there exist some neighborhood $\mathscr{U}_{\lambda_{0}}$ of $\lambda_{0}$, and a finite set $\Sigma$ of points called Landau resonances such that $(H-\lambda)^{-1}$ extends holomorphically to the universal covering of $\mathscr{U}_{\lambda_{0}} \backslash \Sigma$. The Landau resonances are usually branch points of $(H-\lambda)^{-1}$ instead of poles.

For the global extension problem, we have to add to $\Sigma$ a closed set of measure zero $\Sigma_{\infty}$ which corresponds to a complex essential spectrum (see Definition 4.5). Then $(H-\lambda)^{-1}$ extends holomorphically to the universal covering of $\mathscr{U} \backslash \Sigma \cup \Sigma_{\infty}$.

The points of $\Sigma$ are generated by "condensation" of resonances of $H_{p}$, or more geometrically by pinching of some integration contours between some singularities of $\left(H_{p}-\lambda\right)^{-1}$. They are analogous to the Landau singularities of Feynman amplitudes. (See Theorem 4.7.)

$\Sigma_{\infty}$ looks more like essential spectrum in the sense that $\Sigma_{\infty}$ acts as a natural boundary for the extension of $(H-\lambda)^{-1}$ between suitable weighted $L^{2}$ spaces. $\Sigma_{\infty}$ comes in part from the fact that we integrate operator-valued functions and that we have to take care of domain considerations.

To make this remark more clear let us compare $H$ with two-body Schrödinger operators with exponentially decreasing potentials. In the last case the resolvent can be extended meromorphically to a strip $\{\lambda \in \mathbf{C} \mid \operatorname{Im} \lambda>-\alpha\}$ for $\alpha$ depending on the rate of decay of the potential. $\Sigma_{\infty}$ plays a role similar to $\operatorname{Im} \lambda=-\alpha$ in this problem.

Let us remark that since $V$ does not decay at infinity in all directions it is not surprising to obtain more complicated singularities for $(H-\lambda)^{-1}$ than in usual two-body Schrödinger operators.

Under a geometric hypothesis we study the growth and ramification properties of $(H-\lambda)^{-1}$ near $\Sigma$ and prove that $(H-\lambda)^{-1}$ is of finite determination and of moderate growth near the points of $\Sigma$. (See Theorem 4.9.)

In generic cases, we can give an asymptotic expansion for $(H-\lambda)^{-1}$ near a Landau resonance $\lambda_{0} \in \Sigma$ and show that the typical behavior is in $\left(\lambda-\lambda_{0}\right)^{\alpha / 2}$ for $\alpha \in \mathbf{Z}$ or in $\left(\lambda-\lambda_{0}\right)^{\alpha} \log \left(\lambda-\lambda_{0}\right)$ with a leading singularity of finite rank. (See Theorem 4.10.) 
In simple cases, we can associate "resonant functions" to the leading singularities of $(H-\lambda)^{-1}$ at $\lambda_{0}$.

In the last part of the paper we treat the case of a periodic crystal perturbed by impurities. One of the main issues in scattering by non-crystalline surfaces is to know what are the new effects in scattering quantities introduced by the presence of impurities or defects.

We model the impurities by adding to the potential $V$ an additional potential $W$ which is exponentially decreasing in $(x, y)$.

Then we prove (see Theorems 5.1, 5.3) that the effects of impurities on $(H+W-\lambda)^{-1}$ is to add usual poles to the Landau resonances of $(H-\lambda)^{-1}$. This can be of practical significance since the Landau resonances should create logarithmic or square-root singularities on scattering cross-sections, which make resonance shapes very different from the usual Breit-Wigner resonance shape created by a pole.

The plan of the paper is the following:

In Sect. I, we introduce the Floquet-Bloch reduction which will be used in subsequent sections.

In Sect. II, we study the spectral theory of the reduced operators $H_{p}$ using Mourre's commutator method.

In Sect. III, we prove the meromorphic extension in $p$ and $\lambda$ of $\left(H_{p}-\lambda\right)^{-1}$ and give formulas for $\left(H_{p}-\lambda\right)^{-1}$ using Fredholm determinants.

In Sect. IV, we prove the main results of the paper using the results of Sect. III and tools from complex analytic geometry.

In Sect. V, we study the resonances created by the presence of impurities.

To conclude let us mention that the methods used in this paper can be applied without much change to other similar problems: molecular scattering by surfaces, electromagnetic or acoustic scattering by periodic obstacles, resonances for periodic potentials.

We will come back to these problems in a subsequent publication.

\section{Floquet Reduction}

In this section, we recall the method of Floquet-Bloch to reduce the study of a periodic Schrödinger operator on $\mathbf{R}^{m}$ to the study of a family of Schrödinger operators on an $m$-torus. In our case, the Hamiltonian is periodic only in the directions tangent to the surface of the crystal, and the reduced Hamiltonian will live on a cylinder instead of a torus.

On $\mathbf{R}^{m}=\mathbf{R}_{x}^{m-1} \times \mathbf{R}_{y}$, we consider the following Hamiltonian: $H=\left(D_{x}\right)^{2}+$ $\left(D_{y}\right)^{2}+V(x, y)$, where $D_{x_{i}}=(1 / i) \partial_{x_{i}}, D_{y}=(1 / i) \partial_{y}$ and $\left(D_{x}\right)^{2}=\sum_{i=1}^{m-1}\left(D_{x_{i}}\right)^{2}$.

We shall assume that $V$ is a multiplicative potential which is $T$-periodic in the $x$ variable for some lattice $T$ in $\mathbf{R}^{m-1}$, i.e.: $V(x+\tau, y)=V(x, y) \forall \tau \in T$.

We will make in the next sections more precise hypotheses on the local singularities and the decay in $y$ of $V(x, y)$.

We will follow the exposition of Skriganov [Sk] of the Floquet reduction. 
We denote by $T^{*}$ the dual lattice of $T$, which is defined as follows: if $\left(a_{1}, \ldots, a_{m-1}\right)$ is a basis for $T$, a basis for $T^{*}$ is given by the $\left(b_{1}, \ldots, b_{m-1}\right)$ such that $\left\langle a_{i}, b_{j}\right\rangle=2 \pi \delta_{i j}$, where $\langle$,$\rangle is the Euclidean scalar product in \mathbf{R}^{m-1}$.

We denote by $F_{T}$ a fundamental domain of $T, F_{T^{*}}$ a fundamental domain of $T^{*}$, which are chosen to be diffeomorphic to the $n-1$ torus $\Pi^{n-1}$. $\mu_{T}$ (respectively $\mu_{T^{*}}$ ) will be the Lebesgue measure of $F_{T}$ (respectively $F_{T^{*}}$ ).

For $\varphi \in \mathscr{S}\left(\mathbf{R}^{m}\right)$, the Schwartz space of rapidly decreasing $C^{\infty}$ functions, and for $p \in F_{T^{*}}$, we set:

$$
K_{p} \varphi(x, y)=\mu_{T}^{1 / 2} \sum_{\tau \in T} \varphi(x+\tau, y) e^{i\langle p, x+\tau\rangle} .
$$

The sum in (1.1) is convergent because of the rapid decay of $\varphi$, and $K_{p} \varphi(x, y)$ is $T$-periodic in $x$, and satisfies the equations:

$$
K_{p+p^{\prime}} \varphi(x, y)=e^{i\left\langle p^{\prime}, x\right\rangle} K_{p} \varphi(x, y), \text { for } p^{\prime} \in T^{*} .
$$

If we consider $K_{p} \varphi(x, y)$ as a function of $(x, y, p) \in F_{T} \times \mathbf{R} \times F_{T}^{*}$, we see as in [Sk] that the family $K_{p}$ extends as a unitary operator $W_{T}$ :

$$
\begin{aligned}
& L^{2}\left(\mathbf{R}^{n}\right) \rightarrow \mathscr{L}=\int_{F_{T^{*}}}^{\oplus} L^{2}\left(C_{T}\right) d p, \\
& \varphi \mapsto K_{p} \varphi(x, y) .
\end{aligned}
$$

Here $C_{T}$ is the cylinder $F_{T} \times \mathbf{R}$.

Assume now that $V$ is $T$-periodic in $x$ and is relatively bounded with respect to the Laplacian $\Delta=\left(D_{x}\right)^{2}+\left(D_{y}\right)^{2}$ with relative bound strictly less than 1 . Then (see [Sk]), we can decompose $H$ as a direct integral of operators:

$$
W_{T} H W_{T}^{-1}=\int_{F_{T^{*}}}^{\oplus} H_{p} d p \quad \text { where: }
$$

$-H_{p}=\left(D_{x}+p\right)^{2}+\left(D_{y}\right)^{2}+V(x, y)$ with domain $H^{2}\left(C_{T}\right)$.

$-W_{T}^{-1}$ is defined by the following formula:

$$
W_{T}^{-1} \varphi(x, y, p)=(2 \pi)^{-(m-1) / 2} \mu_{T^{*}}^{-1 / 2} \int_{F_{T^{*}}} e^{-i\langle p, x\rangle} \varphi(x, y, p) d p .
$$

We will denote by $K_{p}^{-1}: L^{2}\left(C_{T}\right) \rightarrow L_{\text {loc }}^{2}\left(\mathbf{R}^{m}\right)$ the operator

$$
K_{p}^{-1}: u \mapsto e^{-i\langle p, x\rangle} u(x, y),
$$

where $u(x, y)$ is extended to $\mathbf{R}^{m}$ by $T$-periodicity in $x$.

Taking the usual Sobolev space on the cylinder $C_{T}, H^{2}\left(C_{T}\right)$ as domain for $H_{p}$ means that we consider eigenfunctions of $H_{p}$ ("Bloch waves") which are $T$-periodic in $x$, and in this version of the Floquet reduction, the $p$-dependence of the reduced operator is in the operator and not in his domain as in the usual reduction.

We will denote by $H_{0, p}=\left(D_{x}+p\right)^{2}+\left(D_{y}^{2}\right)$ the free reduced operator with domain $H^{2}\left(C_{T}\right)$.

\section{Spectral Theory of the Reduced Operators}

In this section, we study the spectral theory of $H_{p}$ and $H_{0, p}$. The spectral theory of $H_{0, p}$ is trivial by separation of variables. To get results on the spectrum of $H_{p}$, 
we will use the method of Mourre (see for example [M], [C.F.K.S.]) and construct a conjugate operator for $H_{p}$.

We will assume in this section that $V$ satisfy the following hypotheses:

$$
V\left(H_{0, p}+i\right)^{-1} \text { is compact. }
$$

(H.2) If $A=\frac{1}{2}\left(y . D_{y}+D_{y} . y\right)$, the form $[V, i A]$ defined on $H^{2}\left(C_{T}\right) \cap D(A)$ extends as an operator bounded from $H^{2}\left(C_{T}\right)$ to $H^{-1}\left(C_{T}\right)$ and compact from $H^{2}\left(C_{T}\right)$ to $H^{-2}\left(C_{T}\right)$.

(H.3) The form $[[V, i A], i A]$ defined on $H^{2}\left(C_{T}\right) \cap D(A)$ extends as a bounded operator from $H^{2}\left(C_{T}\right)$ to $H^{-2}\left(C_{T}\right)$.

For example if $V \in C^{2}\left(C_{T}\right), V$ and $y \cdot \nabla_{y} V$ tend to zero when $y$ tends to infinity, and $y^{2} \cdot \nabla_{y}^{2} V$ is bounded, $V$ satisfy $(H . i) i=1,2,3$.

II.A. Spectral Theory of $H_{0, p}$. Let $u \in H^{2}\left(C_{T}\right)$. Since $u$ is $T$ periodic in $x$, we can write:

$$
u(x, y)=\sum_{n \in T^{*}} u_{n}(y) e^{i\langle n, x\rangle}
$$

where

$$
u_{n}(y)=\frac{1}{\mu_{T}^{1 / 2}} \int_{F_{T}} e^{-i\langle n, x\rangle} u(x, y) d x
$$

We have

$$
H_{0, p} u(x, y)=\sum_{n \in T^{*}}\left(D_{y}^{2}+(n+p)^{2}\right) u_{n}(y) e^{i\langle n, x\rangle} .
$$

This gives immediately the spectral decomposition of $H_{0, p}$ : let us denote by $F$ :

$$
\begin{gathered}
L^{2}\left(C_{T}\right) \rightarrow \mathscr{H}=\bigoplus_{T^{*}} L^{2}(\mathbf{R}), \\
u(x, y) \mapsto\left(\hat{u}_{n}(\xi)\right)_{n \in T^{*}},
\end{gathered}
$$

where $\hat{u}_{n}(\xi)=(2 \pi)^{-1 / 2} \int e^{-i y \cdot \xi} u_{n}(y) d y$.

$F$ is obviously unitary from $L^{2}\left(C_{T}\right)$ into $\mathscr{H}$ and we have:

$$
H_{0, p} F^{*}\left(\hat{u}_{n}\right)=F^{*}\left(\left((p+n)^{2}+\xi^{2}\right) \hat{u}_{n}\right) \text {. }
$$

For $p \in F_{T^{*}}$, we will call thresholds of $H_{p}$ the numbers $(n+p)^{2}=\left(n_{1}+p_{1}\right)^{2}+$ $\cdots\left(n_{m-1}+p_{m-1}\right)^{2}$ for $n \in T^{*}$, and denote by $E_{0}(p)=\inf _{n \in T^{*}}\left\{(n+p)^{2}\right\}$.

From the explicit spectral decomposition of $H_{0, p}$, we get immediately:

\section{Proposition 2.1.}

$$
\sigma\left(H_{0, p}\right)=\sigma_{\mathrm{ac}}\left(H_{0, p}\right)=\left[E_{0}(p),+\infty[.\right.
$$

Finally we will write for later use the resolvent kernel of $\left(H_{0, p}-\lambda\right)$ : the kernel $K_{0, p}\left(x, y, x^{\prime}, y^{\prime}, \lambda\right)$ of $\left(H_{0, p}-\lambda\right)^{-1}$ has the following form:

$$
K_{0, p}\left(x, y, x^{\prime}, y^{\prime}, \lambda\right)=\sum_{n \in T^{*}} e^{i\left\langle n, x-x^{\prime}\right\rangle} \frac{1}{2\left(\lambda-(n+p)^{2}\right)^{1 / 2}} e^{i\left(\lambda-(n+p)^{2}\right)^{1 / 2}\left|y-y^{\prime}\right|}
$$

$K_{0, p}$ is bounded from $L^{2}\left(C_{T}\right)$ into $H^{2}\left(C_{T}\right)$ if $\operatorname{Im}\left(\left(\lambda-(n+p)^{2}\right)^{1 / 2}\right)>0 \forall n \in T^{*}$. Equation (2.2) follows directly from the separation of variables used above. 
II.B. Spectral Theory of $H_{p}$. We will prove in this subsection the following theorem:

Theorem 2.2. Under the hypotheses (H.1), (H.2), (H.3), we have

$$
\sigma_{\text {ess }}\left(H_{p}\right)=\left[E_{0}(p),+\infty[,\right.
$$

the eigenvalues of $H_{p}$ can accumulate only at the thresholds $(n+p)^{2}$ for $n \in T^{*}$,

$$
\sigma_{\text {sing }}\left(H_{p}\right)=\varnothing \text {. }
$$

Proof. (2.3) Follows directly from (H.1), Proposition 2.1 and Weyl's theorem on essential spectrum. (cf. [Re-Si]). To prove (2.4), (2.5), we will apply Mourre's commutator method and we refer to the book [C.F.K.S.] for details.

As a conjugate operator we take $A=\frac{1}{2}\left(y \cdot D_{y}+D_{y} \cdot y\right)$. $A$ is the generator of dilations in the $y$ direction, which is the only direction in which classical particles can escape to infinity. Then we have

$$
\left[H_{0, p}, i A\right]=2\left(D_{y}\right)^{2}, \quad\left[H_{p}, i A\right]=2\left(D_{y}\right)^{2}-y \cdot \nabla_{y} V .
$$

We let the reader check that the technical hypotheses on $H, A,[H, i A]$ and $[[H, i A], i A]$ needed to apply Mourre's theorem are satisfied with this choice of $A$, under the hypotheses (H.1), (H.2), (H.3) (see [C.F.K.S.]).

Let now $\Delta \subset\left[E_{0}(p),+\infty[\right.$ be a closed interval not containing any threshold. We will check that $H_{p}$ satisfies a Mourre estimate on $\Delta$.

Let $\chi \in C_{0}^{\infty}(\Delta)$ be a cutoff function with $\chi \equiv 1$ on $\Delta^{\prime} \subset \subset \Delta$. Using that $\left(H_{p+i}\right)^{-1}-\left(H_{0, p+i}\right)^{-1}$ is compact by $(H .1)$, we see that $\chi\left(H_{p}\right)-\chi\left(H_{0, p}\right)$ is compact by a Stone-Weierstrass argument. (H.2) and (2.6) give that:

$$
\chi\left(H_{0, p}\right)\left[H_{p}, i A\right] \chi\left(H_{0, p}\right)=2 \chi\left(H_{0, p}\right)\left(D_{y}\right)^{2} \chi\left(H_{0, p}\right)+K,
$$

where $K$ is a compact operator.

Using the spectral resolution of $H_{0, p}$, we have

$$
F\left(\chi\left(H_{0, p}\right) u\right)_{n}=\hat{u}_{n}(\xi) \times \chi\left(\xi^{2}+(n+p)^{2}\right) .
$$

Since $\Delta$ does not contain any threshold, there exist some $\alpha>0$ such that: $\forall n \in T^{*}$, $\xi \in \mathbf{R}$ such that $\chi\left(\xi^{2}+(n+p)^{2}\right) \neq 0$, we have

This implies that

$$
\xi^{2} \geqq \alpha .
$$

$$
\chi\left(H_{0, p}\right)\left[H_{p}, i A\right] \chi\left(H_{0, p}\right) \geqq 2 \alpha \chi^{2}\left(H_{0, p}\right)+K .
$$

If we replace $\chi\left(H_{0, p}\right)$ by $\chi\left(H_{p}\right)$, we will introduce error terms of the type

$$
\left(\chi\left(H_{0, p}\right)-\chi\left(H_{p}\right)\right)\left[H_{p}, i A\right] \chi\left(H_{0, p}\right)
$$

and

$$
\chi\left(H_{0, p}\right)\left[H_{p}, i A\right]\left(\chi\left(H_{0, p}\right)-\chi\left(H_{p}\right)\right)
$$

which are compact, using (H.2) and (2.6).

So we get

$$
\chi\left(H_{p}\right)\left[H_{p}, i A\right] \chi\left(H_{p}\right) \geqq 2 \alpha \chi^{2}\left(H_{p}\right)+\tilde{K},
$$

where $\tilde{K}$ is compact. 
By composing (2.8) to the left and right by $E_{\Delta^{\prime}}\left(H_{p}\right)$, we prove that $H_{p}$ satisfies a Mourre estimate on $\Delta^{\prime}$, and also on $\Delta$ if we enlarge a little $\Delta$.

Using the theorem of Mourre (see [C.F.K.S.] Theorem 4.7 and Corollary 4.10) we get (2.4) and (2.5).

We make now a few remarks on this theorem:

Remark 2.3. The clinder $C_{T}$ is a special case of a Riemannian manifold with a finite number of cylindrical ends. The spectral theory of the Laplace-Beltrami operator on this kind of manifold (and also on more general ones) has been studied recently by Froese and Hislop (see $[\mathrm{F} . \mathrm{H}],[\mathrm{H}]$ ), and by Guillopé ([G]). In particular Froese and Hislop use Mourre's commutator method to get results on the spectrum.

Remark 2.4. We cannot exclude the existence of embedded eigenvalues as the case of $V(x, y)=V(y)$ shows. In this case, denoting by $\lambda_{k}, k \in \mathbf{N}$ the eigenvalues of $\left(D_{y}\right)^{2}+V(y), H_{p}$ has the eigenvalues $(n+p)^{2}+\lambda_{k}, n \in T^{*}, k \in \mathbf{N}$, with an infinite number of embedded ones. However for a general potential, we expect that these eigenvalues will dissolve in the continuous spectrum due to coupling between different channels, and become resonances.

This mechanism is responsible for resonance creation in atom-surface scattering.

\section{Analytic Extension of the Reduced Resolvent}

In this section we will study the analytic extension in $(p, \lambda)$ of the reduced resolvent $\left(H_{p}-\lambda\right)^{-1}$, when the potential $V$ is exponentially decreasing in the $y$ variable.

We will use a method originally used by Vainberg [Va] in the study of obstacle scattering, which has the advantage of eliminating the continuous spectrum. We will assume in general that $V \in L_{\alpha}^{\infty}\left(C_{T}\right)=\left\{V \mid e^{\alpha\langle y\rangle} V \in L^{\infty}\left(C_{T}\right)\right\}$ for some $\alpha>0$ big enough. Here $\langle y\rangle=\left(1+y^{2}\right)^{1 / 2}$.

This is certainly not the optimal class since we can allow local singularities of $V$ (see Remark 3.4).

We denote by $L_{\alpha}^{2}\left(C_{T}\right)=\left\{u \in L_{\text {loc }}^{2}\left(C_{T}\right) \mid e^{\alpha\langle y\rangle} u \in L^{2}\left(C_{T}\right)\right\} \quad$ and $H_{a}^{1}\left(C_{T}\right)=$ $\left\{u \in H_{\text {loc }}^{1}\left(C_{T}\right) \mid e^{\alpha\langle y\rangle} u \in H^{1}\left(C_{T}\right)\right\}$, for $a \in \mathbf{R}$. We put on these Hilbert spaces their natural norms.

In studying the analytic extension in $(p, \lambda)$ of $\left(H_{p}-\lambda\right)^{-1}$, we must take into consideration the fact that for $p$ fixed, $\left(H_{0, p}-\lambda\right)^{-1}$ (and hence $\left.\left(H_{p}-\lambda\right)^{-1}\right)$ has branch points in $\lambda$ at the thresholds $(n+p)^{2}$ for $n \in T^{*}$ (see (2.2)). When $\lambda$ varies continuously in some bounded open set $\mathscr{U}$ of $\mathbf{C}$, and $p$ in some bounded complex neighborhood $\mathscr{W}$ of $F_{T^{*}}$ in $\mathbf{C}^{m-1}$, only a finite number of the functions $\left(\lambda-(n+p)^{2}\right)^{1 / 2}$ can change their determination. Let us denote by $\mathscr{I} \subset T^{*}$ the set of the $n$ such that $\left(\lambda-(n+p)^{2}\right)^{1 / 2}$ can change of determination, and put $N=\operatorname{Card}(\mathscr{I}), \mathscr{I}=\left\{n_{1}, \ldots, n_{N}\right\}$.

To uniformize the functions $\left(\lambda-(n+p)^{2}\right)^{1 / 2}$ for $n \in \mathscr{I}$, we introduce the following complex analytic set:

$$
\Gamma \subset \mathbf{C}^{N+m}=\left\{\left(p, \lambda, z_{1}, \ldots, z_{N}\right) \in \mathscr{W} \times \mathscr{U} \times \mathbf{C}^{N} \mid z_{i}^{2}=\lambda-\left(n_{i}+p\right)^{2}, i=1, \ldots, N\right\} .
$$

On $\Gamma$ we have a particular region denoted by $\Gamma_{\infty}$, which corresponds to the 
"physical sheet." $\Gamma_{\infty}$ is the subset of $\Gamma$, where $\operatorname{Im} z_{i}>0, i=1, \ldots, N$, and on $\Gamma_{\infty}, \Gamma$ is a smooth submanifold which can be parametrized by $(p, \lambda)$. For $p \in F_{T^{*}}, \lambda \in \mathscr{U}$, $\operatorname{Im} \lambda>0$, we can write the kernel of $\left(H_{0, p}-\lambda\right)^{-1}$ as the restriction to $\Gamma_{\infty}$ of the function:

$$
\begin{aligned}
K_{0}\left(x, y, x^{\prime}, y^{\prime}, p, \lambda, z_{1}, \ldots, z_{N}\right)= & \sum_{j=1}^{N} e^{i\left\langle n_{j}, x-x^{\prime}\right\rangle} \frac{1}{2 z_{j}} e^{i z_{j}\left|y-y^{\prime}\right|} \\
& +\sum_{n \in T^{*} \backslash \mathscr{G}} e^{i\left\langle n, x-x^{\prime}\right\rangle} \frac{1}{2\left(\lambda-(n+p)^{2}\right)^{1 / 2}} e^{i\left(\lambda-(n+p)^{2}\right)^{2 / 2}\left|y-y^{\prime}\right|} .
\end{aligned}
$$

We remark that $\Gamma_{\infty}$ is the natural region where $K_{0}$ is the kernel of an operator $K_{0}(p, \lambda, z)$ which is bounded on $L^{2}\left(C_{T}\right)$. We denote for simplicity by $z$ the $N$-uple $\left(z_{1}, \ldots, z_{N}\right)$, and let $z$ vary in some set $Z$ of the type: $Z=\left\{z \in \mathbf{C}^{N} \mid \operatorname{Im} z_{i} \geqq-\varepsilon\right\}$ for some $\varepsilon>0$. We have the following Proposition:

Proposition 3.1. For $a>\varepsilon, K_{0}(p, \lambda, z)$ extends meromorphically in $(p, \lambda, z) \in \mathscr{W} \times \mathscr{U} \times Z$ as a bounded operator from $L_{a}^{2}\left(C_{T}\right)$ into $H_{-a}^{1}\left(C_{T}\right)$ with singularities on $z_{i}=0$, $i=1, \ldots, N$.

Proof. To study the boundedness of $K_{0}(p, \lambda, z)$ from $L_{a}^{2}\left(C_{T}\right)$ into $H_{-a}^{1}\left(C_{T}\right)$, we have to investigate the following reduced kernel:

$$
\begin{aligned}
K_{\mathrm{red}}\left(x, y, x^{\prime}, y^{\prime}, p, \lambda, z\right)= & \sum_{j=1}^{N} e^{i\left\langle n_{j}, x-x^{\prime}\right\rangle} \frac{1}{2 z_{j}} e^{i z_{j}\left|y-y^{\prime}\right|-a\langle y\rangle-a\left\langle y^{\prime}\right\rangle} \\
& +\sum_{n \in T^{*} \backslash \mathscr{G}} e^{i\left\langle n, x-x^{\prime}\right\rangle} \frac{1}{2\left(\lambda-(n+p)^{2}\right)^{1 / 2}} e^{i\left(\lambda-(n+p)^{2}\right)^{1 / 2}\left|y-y^{\prime}\right|-a\langle y\rangle-a\left\langle y^{\prime}\right\rangle} .
\end{aligned}
$$

For $(p, \lambda) \in \mathscr{W} \times \mathscr{U}, n \in T^{*} \backslash \mathscr{I}$, we have:

$$
\left(\lambda-(n+p)^{2}\right)^{1 / 2}=i\left(n^{2}\right)^{1 / 2}\left(1+\frac{2 n \cdot p}{n^{2}}+\frac{p^{2}}{n^{2}}-\frac{\lambda}{n^{2}}\right)^{1 / 2}=i|n|\left(1+0\left(\frac{1}{|n|}\right)\right),
$$

uniformly for $(p, \lambda) \in \mathscr{W} \times \mathscr{U}$.

We use here the fact that $\operatorname{Im}\left(\left(\lambda-(n+p)^{2}\right)^{1 / 2}\right)>0$, for $(p, \lambda) \in \mathscr{W} \times \mathscr{U}, n \notin \mathscr{I}$.

Finally we have:

$$
\left(\lambda-(n+p)^{2}\right)^{1 / 2}=i|n|+O(1) \text { for }(p, \lambda) \in \mathscr{W} \times \mathscr{U}, \quad n \notin \mathscr{I} .
$$

We can now prove the Proposition: since $K_{0}$ splits on the direct sum decomposition $L^{2}\left(C_{T}\right)=\bigoplus_{n \in T^{*}} e^{i\langle n, x\rangle} \otimes L^{2}(\mathbf{R})$, we only have to estimate the following operators:

$$
M_{n}: u(y) \mapsto e^{-i\langle n, x\rangle} M\left(e^{i\langle n, x\rangle} u(y)\right)
$$

for $M=K_{\mathrm{red}}, \partial_{y} K_{\mathrm{red}}, \partial_{x_{i}} K_{\mathrm{red}}, i=1, \ldots, m-1$, as operators on $L^{2}(\mathbf{R})$. For $M=K_{\mathrm{red}}$, we get an operator $M_{n}$ with kernel:

$$
\begin{aligned}
& \frac{1}{2 z_{j}} e^{i z_{j}\left|y-y^{\prime}\right|-a\langle y\rangle-a\left\langle y^{\prime}\right\rangle} \quad \text { if } n=n_{j}, \\
& \frac{1}{2\left(\lambda-(n+p)^{2}\right)^{1 / 2}} e^{i\left(\lambda-(n+p)^{2}\right)^{1 / 2}\left|y-y^{\prime}\right|-a\langle y\rangle-a\left\langle y^{\prime}\right\rangle} \text { if } n \notin \mathscr{I} .
\end{aligned}
$$


Using (3.2) and the criterion of Schur, we get that $M_{n}$ is bounded on $L^{2}(\mathbf{R})$ with norm $\left\|M_{n}\right\| \leqq C$ uniformly in $n$, provided we take $a>\varepsilon, z_{i} \neq 0$. For $M=\partial_{y} K_{\text {red }}$ or $\partial_{x_{i}} K_{\text {red }}$, we get an operator $M_{n}$ with kernel equal respectively to (if $n=n_{j}$ ):

$$
\frac{1}{2} \frac{y-y^{\prime}}{\left|y-y^{\prime}\right|} e^{i z j\left|y-y^{\prime}\right|-a\langle y\rangle-a\left\langle y^{\prime}\right\rangle}
$$

and

$$
\frac{n_{i j}}{2 z_{j}} e^{i z_{j}\left|y-y^{\prime}\right|-a\langle y\rangle-a\left\langle y^{\prime}\right\rangle} .
$$

They satisfy the same estimates as above. The case $n \notin \mathscr{I}$ is similar, which proves the Proposition.

We will need also the following lemma:

Lemma 3.2. The residues of $K_{0}(p, \lambda, z)$ at $z_{j}=0$ are finite rank operators.

Proof. The singularity in $z_{j}^{-1}$ at a threshold $\left(n_{j}+p\right)^{2}$ comes from the singularity of $\left(D_{y}^{2}-z_{j}^{2}\right)^{-1}$, which has the kernel: $e^{i z_{j}\left|y-y^{\prime}\right|} / 2 z_{j}$. We will show how to isolate this singularity for $\left(D_{y}^{2}-z_{j}^{2}\right)^{-1}$ and then come back to $K_{0}(p, \lambda, z)$.

We can write:

$$
e^{i z_{j}\left|y-y^{\prime}\right|}=1+\int_{0}^{1} i z_{j}\left|y-y^{\prime}\right| e^{i z_{j}\left|y-y^{\prime}\right| t} d t .
$$

Using (3.3), we can write:

$$
\left(D_{y}^{2}-z_{j}^{2}\right)^{-1} u=\frac{1}{2 z_{j}} \int u\left(y^{\prime}\right) d y^{\prime}+M\left(z_{j}\right) u,
$$

where by the same arguments as in the proof of Proposition 3.1, we see that $M\left(z_{j}\right)$ is holomorphic in $z_{j}$ as a bounded operator between $L_{a}^{2}(\mathbf{R})$ and $H_{-a}^{1}(\mathbf{R})$, for $a>-\operatorname{Inf}\left(\operatorname{Im} z_{j}\right)$.

We can now come back to $K_{0}(p, \lambda, z)$ : for $u \in L_{a}^{2}\left(C_{T}\right)$, if we put

$$
\pi_{j} u(x, y)=e^{i\left\langle n_{j}, x\right\rangle} \mu_{T}^{-1} \int_{F_{T}} e^{-i\left\langle n_{j}, x\right\rangle} u(x, y) d x,
$$

$\pi_{j}$ is bounded on $L_{a}^{2}\left(C_{T}\right)$.

We see directly by looking at expression (3.1) for $K_{0}(p, \lambda, z)$, that $K_{0}(p, \lambda, z)\left(1-\pi_{j}\right)$ has no singularities on $z_{j}=0$, as a bounded operator from $L_{a}^{2}\left(C_{T}\right)$ into $H_{-a}^{1}\left(C_{T}\right)$.

On the other hand:

$$
K_{0}(p, \lambda, z) \pi_{j} u=e^{i\left\langle n_{j}, x\right\rangle}\left(D_{y}^{2}-z_{j}^{2}\right)^{-1} e^{-i\left\langle n_{j}, x\right\rangle} \pi_{j} u=\frac{\tilde{\pi}_{j}}{z_{j}} u+\tilde{M}(p, \lambda, z) u,
$$

where $\tilde{M}(p, \lambda, z)$ is holomorphic near $z_{j}=0$ and $\tilde{\pi}_{j}$ is a rank one operator

$$
\tilde{\pi}_{j} u=e^{i\left\langle n_{j}, x\right\rangle}\left(2 \mu_{T}\right)^{-1} \int_{C_{T}} e^{-i\left\langle n_{j}, x\right\rangle} u(x, y) d x d y .
$$

This proves the lemma. 
We need one more result before proving Theorem 3.5. Let us denote by $C(Z)$ the constant $-\operatorname{Inf}_{z \in Z}\left(\operatorname{Im} z_{i}\right)$. We have:

Proposition 3.3. Assume that $V \in L_{\alpha}^{\infty}\left(C_{T}\right)$ for $\alpha>2 C(Z)$. Then $V K_{0}(p, \lambda, z)$ belongs to the Schatten class $\mathscr{F}_{k}$ on $L_{a}^{2}\left(C_{T}\right)$, for $a>C(Z),(p, \lambda, z) \in \mathscr{W} \times \mathscr{U} \times Z, z_{i} \neq 0, k>m$.

Proof. It clearly suffices to prove that $e^{-\alpha\langle y\rangle} K_{0}(p, \lambda, z)$ is in $\mathscr{F}_{k}$. Let us first remark that if $\alpha>2 C(Z)+\varepsilon_{0}$ for $\varepsilon_{0}>0$, we have that $e^{-\left(\alpha-\varepsilon_{0}\right)\langle y\rangle} K_{0}(p, \lambda, z)$ is bounded from $L_{a}^{2}\left(C_{T}\right)$ into $H_{a}^{1}\left(C_{T}\right)$, for $a>C(Z)$. This means that $e^{\left(a-\alpha+\varepsilon_{0}\right)\langle y\rangle} K_{0}(p, \lambda, z) e^{-a\langle y\rangle}$ is bounded from $L^{2}\left(C_{T}\right)$ into $H^{1}\left(C_{T}\right)$, or that

$$
(1+|\Delta|)^{1 / 2} e^{\left(a-\alpha+\varepsilon_{0}\right)\langle y\rangle} K_{0}(p, \lambda, z) e^{-a\langle y\rangle}
$$

is $L^{2}\left(C_{T}\right)$ bounded for $(p, \lambda, z) \in \mathscr{W} \times \mathscr{U} \times Z, z_{i} \neq 0$.

To prove the Proposition, it suffices to show that:

$$
e^{-\varepsilon_{0}\langle y\rangle}(1+|\Delta|)^{-1 / 2} \quad \text { belongs to } \mathscr{F}_{k} \text { for } k>m \text { on } L^{2}\left(C_{T}\right) .
$$

Using Theorem 4.1 of Simon [Si], which can be easily adapted to our case, we see that (3.4) holds, which proves the Proposition.

Remark 3.4. In order to include local singularities of $V$, we can introduce the space $L_{\alpha}^{k}\left(C_{T}\right)=\left\{V \in L_{\text {loc }}^{k}\left(C_{T}\right) \mid e^{\alpha\langle y\rangle} V \in L^{k}\left(C_{T}\right)\right\}$. If $V \in L_{\alpha}^{k}\left(C_{T}\right)$ for $k>m, V$ belongs to the Stummel class $S_{m}$ (see [C.F.K.S.]) so $H$ and $H_{p}$ are self adjoint with domains $H^{2}\left(\mathbf{R}^{m}\right)$ and $H^{2}\left(C_{T}\right)$ respectively. Using Theorem 4.1 of [Si] we see that Proposition 3.3 still holds if $V \in L_{\alpha}^{k}\left(C_{T}\right), k>m, \alpha>2 C(Z)$.

Theorem 3.5. Assume that $V$ belongs to $L_{\alpha}^{k}\left(C_{T}\right)$ for $k>m, \alpha>2 C(Z)$. Then $\left(1+V K_{0}(p, \lambda, z)\right)^{-1}$ can be written for $(p, \lambda, z) \in \mathscr{W} \times \mathscr{U} \times Z$ as

$$
\left(1-V K_{0}(p, \lambda, z)\right)^{-1}=\frac{D(p, \lambda, z)}{f(p, \lambda, z)},
$$

where $D$ and $f$ are holomorphic in $(p, \lambda, z) \in \mathscr{W} \times \mathscr{U} \times Z$ as a bounded operator on $L_{a}^{2}\left(C_{T}\right)$ and as a function respectively, for $a>C(Z)$.

Proof. From Lemma 3.2 and his proof we see that $K(p, \lambda, z)=V K_{0}(p, \lambda, z)$ can be written as:

$$
K(p, \lambda, z)=M(p, \lambda, z)+\sum_{j=1}^{N} \frac{\pi_{j}}{z_{j}}=M(p, \lambda, z)+R(p, \lambda, z)
$$

where

$-M(p, \lambda, z)$ is holomorphic in $(p, \lambda, z) \in \mathscr{W} \times \mathscr{U} \times Z$ and satisfies the properties of Proposition 3.3.

$-\pi_{j} j=1, \ldots, N$ are rank one operators on $L_{a}^{2}\left(C_{T}\right)$.

We will write:

$$
(1+K)^{-1}=(1+M)^{-1}\left(1+R(1+M)^{-1}\right)^{-1} .
$$

We first consider $(1+M(p, \lambda, z))^{-1}$. We will use the theory of regularized determinants for operators in $\mathscr{F}_{k}$. We follow the exposition of [Si] paragraph 9 . We 
consider for $N_{0} \geqq m+1$ :

$$
R(M)=(1+M) \exp \left(-\sum_{1}^{N_{0}-1}(-M)^{k} / k\right)-1 .
$$

$R(M)$ is trace class with $\|R(M)\|_{T_{r}} \leqq C\|M\|_{N_{0}}$, where \|\|$_{T_{r}}$ is the trace norm and $\|\quad\|_{N_{0}}$ is the norm in $\mathscr{F}_{N_{0}}$.

Then $1+M$ is invertible if and only if the usual Fredholm determinant $\operatorname{det}(\mathbf{1}+R(M))$ is non-zero, and:

$$
(1+M)^{-1}=(1+R(M))^{-1} \exp \left(-\sum_{1}^{N_{0}-1}(-M)^{k} / k\right) .
$$

$R(M)$ is holomorphic in $(p, \lambda, z) \in \mathscr{W} \times \mathscr{U} \times Z$ as a bounded operator in $L_{a}^{2}\left(C_{T}\right)$ and belongs to $\mathscr{F}_{1}$ with $\|R(M)\|_{T_{r}} \leqq C$ uniformly in $(p, \lambda, z)$. So we are reduced to the case when $M$ is trace class. The Fredholm determinant of $M$ is then: $\operatorname{det}(1+M)=$ $\sum_{m=0}^{\infty} D_{0, m}(M)$, where $D_{0, m}(M)$ is a polynomial expression of the $\operatorname{tr}\left(M^{k}\right)$ for $1 \leqq k \leqq m$, satisfying:

$$
\left|D_{0, m}(M)\right| \leqq\left(\frac{c}{m}\right)^{m / 2}\|M\|_{T_{r}}^{m} .
$$

(see $[\mathrm{Si}])$. To show that $\operatorname{det}(1+M)$ is holomorphic in $(p, \lambda, z)$, it suffices to show that $D_{0, m}(M)$ (i.e. $\left.\operatorname{Tr}\left(M^{k}\right)\right)$ is holomorphic in $(p, \lambda, z)$. Then $\operatorname{det}(1+M)$ will be holomorphic as a uniformly convergent series of holomorphic functions.

It is easy to check that $\operatorname{Tr}\left(M^{k}\right)$ is holomorphic in $(p, \lambda, z)$, which shows that $\operatorname{det}(1+M)$ is a holomorphic function.

If $\operatorname{det}(1+M) \neq 0,(1+M)^{-1}$ can be written as: $D_{1}(p, \lambda, z) / \operatorname{det}(1+M(p, \lambda, z))$, where $D_{1}(p, \lambda, z)$ is of the form:

$$
D_{1}(p, \lambda, z)=\sum_{0}^{+\infty} D_{1, m}(M)
$$

and $D_{1, m}(M)$ is a polynomial expression of the $M^{k}, \operatorname{Tr}\left(M^{k}\right)$ for $1 \leqq k \leqq m$, satisfying:

$$
\left\|D_{1, m}(M)\right\| \leqq C^{m} \frac{1}{m^{m / 2}}\|M\|_{T_{r}}^{m} \quad(\text { see }[\mathrm{Si}]) .
$$

As before, we prove that $D_{1, m}(M)$ and then $D_{1}(p, \lambda, z)$ are holomorphic for $(p, \lambda, z) \in \mathscr{W} \times \mathscr{U} \times Z$. So we can write $(1+M)^{-1}$ as: $D_{1}(p, \lambda, z) / f_{1}(p, \lambda, z)$, and using the estimates in the proof of Proposition 3.1, it is easy to check that $(1+M(p, \lambda, z))$ is invertible for $p \in \mathscr{W}, \operatorname{Im} \lambda \gg 1, \operatorname{Im} z_{i} \gg 1$ which proves that $f_{1}(p, \lambda, z) \neq \equiv$.

From (3.6), we see that to invert $(1+K)^{-1}$, it remains to invert $1+\pi(z) \dot{D}_{1}(p, \lambda, z) /$ $g(z) f_{1}(p, \lambda, z)$, where $\pi(z)=\sum_{j=1}^{N} \pi_{j} z_{1} \ldots z_{j-1} z_{j+1} \ldots z_{N}, \quad$ and $\quad g(z)=z_{1} \ldots z_{N}$. $\pi(z) D_{1}(p, \lambda, z) / g(z) f_{1}(p, \lambda, z)$ can be written as $\tilde{\pi}(p, \lambda, z) / h(p, \lambda, z)$, where $h=g \times f_{1}$, and $\tilde{\pi}$ has a fixed image $F$ of finite dimension.

Now to solve

$$
u+\frac{\tilde{\pi}}{h} u=v
$$


we try $u=\left(1-\pi_{0}\right) r+r$, where $\pi_{0}$ is the orthogonal projection in $F$ and $r \in F$. We get:

$$
r+\frac{\tilde{\pi}}{h} r=\pi_{0} v-\frac{\tilde{\pi}}{h}\left(1-\pi_{0}\right) v=E(p, \lambda, z) v .
$$

The equation in the finite dimensional space $F: r+(\tilde{\pi} / h) r=f$ can be solved by: $r=\tilde{D}(p, \lambda, z) / \tilde{h}(p, \lambda, z)$, where $\widetilde{D}$ and $\tilde{h}$ are holomorphic in $(p, \lambda, z)$ as an operator and as a function respectively.

This follows from finite dimensional matrix theory.

Finally we solve (3.7) by

$$
u=\left(1-\pi_{0}\right) v+\frac{\tilde{D}(p, \lambda, z)}{\tilde{h}(p, \lambda, z)} E(p, \lambda, z) v .
$$

Putting together (3.6), (3.9), we get that $(1+K(p, \lambda, z))^{-1}$ can be written as: $D(p, \lambda, z) / f(p, \lambda, z)$ where $D$ and $f$ are holomorphic in $(p, \lambda, z) \in \mathscr{W} \times \mathscr{U} \times Z$ as an operator on $L_{a}^{2}\left(C_{T}\right)$ and as a function respectively. This proves the theorem.

\section{Analytic Extension of the Total Resolvent}

In this section we will prove the existence of an analytic extension for the total resolvent $(H-\lambda)^{-1}$. As explained in the introduction, the singularities of $(H-\lambda)^{-1}$ are different when one considers the local extension of $(H-\lambda)^{-1}$ in a small neighborhood of $\lambda_{0} \in \mathbf{R}$, and when one considers the global extension of $(H-\lambda)^{-1}$ to $\mathscr{U}$, where $\mathscr{U}$ is a bounded set in $\mathbf{C}$ as in Sect. III.

In the local case, $(H-\lambda)^{-1}$ extends holomorphically as a bounded operator between some weighted $L^{2}$ spaces to the universal covering of $\mathscr{U}_{\lambda_{0}} \backslash \Sigma$, where $\mathscr{U}_{\lambda_{0}}$ is a neighborhood of $\lambda_{0}$ and $\Sigma$ is a discrete set of points in $\mathscr{U}$, called Landau resonances.

In the global case in addition to $\Sigma,(H-\lambda)^{-1}$ can have singularities on a closed set of measure zero $\Sigma_{\infty}$ which correspond to a kind of complex essential spectrum. We will study some properties of $(H-\lambda)^{-1}$ like finite determination and moderate growth under some additional hypotheses near the points of $\Sigma$. The points in $\Sigma$ are analogous to Landau singularities in quantum field theory. Using results of Leray and Pham we will then study the behavior of $(H-\lambda)^{-1}$ near generic points of $\Sigma$ and get asymptotic expansions which show that in general a Landau resonance is a branch point of $(H-\lambda)^{-1}$ rather than a pole.

In some cases it is even possible to associate to a Landau resonance a kind of "generalized resonant state."

We start by proving some formulas.

From the discussion in Sect. I, we see that for $\operatorname{Im} \lambda>0,(H-\lambda)^{-1}$ can be written as:

$$
\begin{gathered}
\text { for } u \in C_{0}^{\infty}\left(\mathbf{R}^{m}\right): \\
(H-\lambda)^{-1} u=C(m, T) \int_{F_{T^{*}}} e^{-\imath\langle p, x\rangle}\left(H_{p}-\lambda\right)^{-1}\left(\sum_{\tau \in T} u(x+\tau, y) e^{i\langle p, x+\tau\rangle}\right) d p,
\end{gathered}
$$

where $C(m, T)=(2 \pi)^{-(m-1) / 2} \mu_{T}^{1 / 2} \mu_{T^{*}}^{-1 / 2}$. 
Using the second resolvent formula, we have:

$$
\left(H_{p}-\lambda\right)^{-1}=\left(H_{0, p}-\lambda\right)^{-1}\left(1+V\left(H_{0, p}-\lambda\right)^{-1}\right)^{-1}, \quad \text { for } \operatorname{Im} \lambda>0, \quad p \in F_{T^{*}} .
$$

On the other hand, $\left(H_{0, p}-\lambda\right)^{-1}$ is the restriction to $\Gamma_{\infty}$ of the operator $K_{0}(p, \lambda, z)$ : if we denote for $p \in F_{T^{*}}, \operatorname{Im} \lambda>0$, by $z_{i}(p, \lambda)$ the determination of $\left(\lambda-\left(n_{i}+p\right)^{2}\right)^{1 / 2}$ with positive imaginary part, we have: $\left(H_{0, p}-\lambda\right)^{-1}=K_{0}(p, \lambda, z(p, \lambda))$.

From Theorem 3.5, we can rewrite (4.1) as:

$$
\begin{aligned}
(H-\lambda)^{-1} u & =C(m, T) \int_{F_{T^{*}}} e^{-i\langle p, x\rangle} \frac{\tilde{D}(p, \lambda, z(p, \lambda))}{f(p, \lambda, z(p, \lambda))}\left(\sum_{\tau \in T} u(x+\tau, y) e^{i\langle p, x+\tau\rangle}\right) d p \\
& =C(m, T) \int_{F_{T^{*}}} \frac{M(p, \lambda, z,(p, \lambda))}{f(p, \lambda, z(p, \lambda))} u d p .
\end{aligned}
$$

For $a \in \mathbf{R}$, we denote by $L_{a}^{2}\left(\mathbf{R}^{m}\right)$ the space

$$
\left\{u \in L_{\mathrm{loc}}^{2}\left(\mathbf{R}^{m}\right) \mid e^{a(\langle x\rangle+\langle y\rangle)} u \in L^{2}\left(\mathbf{R}^{m}\right)\right\}
$$

and by $H_{a}^{1}\left(\mathbf{R}^{m}\right)$ the space

$$
\left\{u \in H_{\mathrm{loc}}^{1}\left(\mathbf{R}^{m}\right) \mid e^{a(\langle x\rangle+\langle y\rangle)} u \in H^{1}\left(\mathbf{R}^{m}\right)\right\} .
$$

$L_{a}^{2}\left(\mathbf{R}^{m}\right)$ and $H_{a}^{1}\left(\mathbf{R}^{m}\right)$ are Hilbert spaces with their natural norms. We have the following Proposition:

Proposition 4.1. If $V \in L_{\alpha}^{k}\left(C_{T}\right)$ for $k>m, \alpha>2\left(C(Z)+\sup |\operatorname{Im} p|_{p \in \mathscr{W}}\right), M(p, \lambda, z)$ extends holomorphically to $(p, \lambda, z) \in \mathscr{W} \times \mathscr{U} \times Z$ as a bounded operator from $L_{a}^{2}\left(\mathbf{R}^{m}\right)$ into $H_{-a}^{1}\left(\mathbf{R}^{m}\right)$ for $a>\left(C(Z)+\sup |\operatorname{Im} p|_{p \in \mathscr{W}}\right)$.

Proof. Using Theorem 3.5, we see that it suffices to prove that $K_{p}$ (defined in (1.1)) extends holomorphically in $p$ as a bounded operator from $L_{a}^{2}\left(\mathbf{R}^{m}\right)$ into $L_{a}^{2}\left(C_{T}\right)$ and that $\left(K_{p}\right)^{-1}$ (defined in (1.4)) extends holomorphically in $p$ as a bounded operator from $H_{-a}^{1}\left(C_{T}\right)$ into $H_{-a}^{1}\left(\mathbf{R}^{m}\right)$. If $a>C \sup |\operatorname{Im} p|_{p \in \mathscr{W}}$ such that $\left|e^{-a\langle x\rangle} e^{i\langle p, x\rangle}\right| \leqq e^{-\varepsilon\langle x\rangle}$ for $p \in \mathscr{W}$, it is clear that $K_{p}$ is uniformly bounded from $L_{a}^{2}\left(\mathbf{R}^{m}\right)$ into $L_{a}^{2}\left(C_{T}\right)$. Consider now the function $e^{-i\langle p, x\rangle} u(x, y)$ for $u \in H_{-a}^{1}\left(C_{T}\right)$ extended by periodicity in $x$ as a function in $H_{\mathrm{loc}}^{1}\left(\mathbf{R}^{m}\right)$.

We have to estimate:

$$
\left\|e^{-a(\langle x\rangle+\langle y\rangle)} e^{-i\langle p, x\rangle} u(x, y)\right\|_{L^{2}\left(\mathbf{R}^{m}\right)}^{2}=\sum_{\tau \in T^{*}}\left\|e^{-a(\langle x+\tau\rangle+\langle y\rangle)} e^{-i\langle p, x+\tau\rangle} u(x, y)\right\|_{L^{2}\left(F_{T}\right)}^{2} .
$$

If $a>C \sup |\operatorname{Im} p|_{p \in \mathscr{W}}$ we have for $p \in \mathscr{W}, x \in F_{T}:\left|e^{-a\langle x+\tau\rangle} e^{-i\langle p, x+\tau\rangle}\right| \leqq C e^{-\varepsilon|\tau|}$ for some $\varepsilon>0$.

So we get a convergent sequence in (4.3) and we have:

$$
\left\|e^{-l\langle p, \lambda\rangle} u\right\|_{L_{-a}^{2}}\left(\mathbf{R}^{m}\right) \leqq C\|u\|_{L_{-a}^{2}\left(C_{T}\right)} .
$$

We can estimate the derivatives of $e^{-\imath\langle p, x\rangle} u$ in the same way, which proves the Proposition.

We now use Cauchy's formula in order to eliminate the multivalued functions $z_{i}(p, \lambda)$. By making a linear change of coordinates in $p$, we may assume that $F_{T^{*}}=[-1,1]^{m-1}$. 
We will consider the operator $(H-\lambda)^{-1}$ for $\lambda$ near some point $\lambda_{0} \in \mathscr{U} \cap\{\operatorname{Im} \lambda>0\}$. Since $f(p, \lambda, z(p, \lambda)) \neq 0$ for $p \in F_{T^{*}}, \operatorname{Im} \lambda>0$, we can find some $\varepsilon>0$ small enough such that if $p$ is in $F_{T^{*}}, \lambda$ in a small neighborhood of $\lambda_{0}$, and $|z-z(p, \lambda)| \leqq \varepsilon$, we have $f(p, \lambda, z) \neq 0$, and such that $z \in Z$ if $|z-z(p, \lambda)| \leqq \varepsilon$. Let $\gamma_{i}(p)$ be the circle of center $\left(\lambda_{0}-\left(n_{i}+p\right)^{2}\right)^{1 / 2}$ and of radius $\varepsilon / 2$. By Cauchy formula, we have, since $f(p, \lambda, z) \neq 0$ if $|z-z(p, \lambda)| \leqq \varepsilon$ :

$$
\frac{M(p, \lambda, z(p, \lambda))}{f(p, \lambda, z(p, \lambda))}=\frac{1}{i \pi} \int_{\gamma_{1}(p)} \frac{M\left(p, \lambda, z_{1}, z_{2}(p, \lambda), \ldots, z_{N}(p, \lambda)\right)}{f\left(p, \lambda, z_{1}, z_{2}(p, \lambda), \ldots, z_{N}(p, \lambda)\right)} \frac{\left(\lambda-\left(n_{1}+p\right)^{2}\right)^{1 / 2}}{z_{1}^{2}-\lambda+\left(n_{1}+p\right)^{2}} d z_{1} .
$$

By iterating this process, we can write $(H-\lambda)^{-1}$ as follows: Let $\Delta$ be the $m+N-1$ dimensional cycle which is the image of $[-1,1]^{m-1} \times T^{N}$ (here $T^{N}$ is the $N$-torus) under the map: $(p, \omega) \rightarrow\left(p, z\left(p, z\left(p, \lambda_{0}\right)+(\varepsilon / 2) \omega\right) \in \mathbf{C}^{m-1} \times \mathbf{C}^{N}\right.$. Then we have for $\lambda$ near $\lambda_{0}$ :

$$
(H-\lambda)^{-1}=C(m, T)(i \pi)^{-N} \int_{\Delta} \frac{M(p, \lambda, z)}{f(p, \lambda, z)} \prod_{i=1}^{N}\left(\lambda-\left(n_{i}+p\right)^{2}\right)^{1 / 2}\left(z_{i}^{2}-\lambda+\left(n_{i}+p\right)^{2}\right)^{-1} d z d p .
$$

So we have written $(H-\lambda)^{-1}$ for $\lambda$ near $\lambda_{0}$ as an integral on $\Delta$ of an operator valued function $G(p, \lambda, z)$ which is holomorphic in $(p, \lambda, z)$ on the universal covering $(\mathscr{W} \times \mathscr{U} \times Z \backslash S)^{*}$ of $\mathscr{W} \times \mathscr{U} \times Z \backslash S$, where $S$ is the analytic set defined by the equations:

$$
\begin{gathered}
S=\{(p, \lambda, z) \in \mathscr{W} \times \mathscr{U} \times Z \mid f(p, \lambda, z)=0 \\
\text { or } \left.\lambda-\left(n_{i}+p\right)^{2}=0 \text { or } z_{i}-\lambda+\left(n_{i}+p\right)^{2}=0 \text { for } 1 \leqq i \leqq N\right\} .
\end{gathered}
$$

It is well known that if $G(p, \lambda, z)$ is a usual holomorphic function on $(\mathscr{W} \times \mathscr{U} \times Z \backslash S)^{*}$ the singularities of $\int_{\Delta} G(p, \lambda, z) d z_{1} \wedge \cdots \wedge d z_{N} \wedge d p_{1} \wedge \cdots \wedge d p_{m-1}$ are much smaller than the crude guess

$$
\S_{\text {sing }}=\{\lambda \mid \exists(p, z) \in \Delta \text { such that }(p, \lambda, z) \in S\} .
$$

(Note that this guess would produce "lines" of singularities if $m=2$ and "balls" of singularities if $m=3$.)

Indeed this integral is holomorphic as long as the integration cycle can be deformed continuously avoiding the set $S$. This is the basis of the analysis of Landau singularities in quantum field theory (see for example [F.F.L.P], [P], [B.P]), of ramification of holomorphic integrals (see $[\mathrm{V}],[\mathrm{L}],[\mathrm{K}]$ ) and of functions of the Nilsson class (see $[\mathrm{Me}]$ ).

The singularities of $(H-\lambda)^{-1}$ will come from the following three types of obstructions:

a) end point singularities: a boundary of the integration cycle is tangent to $S$.

b) pinching singularities: the cycle is pinched between some strata of $S$.

c) pinching singularities at infinity: the cycle is pinched between some stratum of $S$ and the infinity or the boundary of the holomorphy domain of $G(p, \lambda, z)$.

A problem very similar to ours (with usual holomorphic functions) has been studied by Kobayashi [K] in relation to singularities of solutions of Cauchy 
problems in the complex domain. We will follow the exposition of Kobayashi. We introduce now some notation.

We put: $Y=\mathscr{W} \times \mathscr{U} \times Z \backslash S, F_{i}^{ \pm}=\left\{(p, z) \in \mathscr{W} \times Z \mid p_{i}= \pm 1\right\}$ for $i=1, \ldots, m-1$,

$$
F=\bigcup_{+,-i} F_{i}^{ \pm}, \tilde{F}_{i}^{ \pm}=F_{i}^{ \pm} \cap \pi_{p, z}(Y), \quad \tilde{F}=F \cap \pi_{p, z}(Y)
$$

where $\pi_{p, z}$ is the projection $(p, \lambda, z) \mapsto(p, z)$, and finally $\tilde{S}$ the analytic set $S \cup(F \times \mathscr{U})$.

If $\pi:(p, \lambda, z) \mapsto \lambda$ is the other projection, we will denote by $A_{\lambda}$ the fiber $\pi^{-1}(\lambda) \cap A$ for $\lambda \in \mathscr{U}, A$ some set in $\mathscr{W} \times \mathscr{U} \times Z$. To describe obstruction c), we will have to replace the domain $\mathscr{W} \times Z$ of the fiber variables $(p, z)$ by a smaller domain having a real analytic boundary. To this end, we consider the function

$$
\delta(p, z)=C_{0} \sum_{i=1}^{m-1}\left(\operatorname{Im} p_{i}\right)^{2}+C_{1}\left(\sum_{i=1}^{N}\left(\operatorname{Im} z_{i}+C(Z)\right)^{2}\right)^{-1}+C_{2} \sum_{i=1}^{N}\left(\operatorname{Im} z_{i}\right)^{2},
$$

where the constants $C_{0}, C_{2}$ have to be chosen large enough and $C_{1}$ small enough.

We will replace $\mathscr{W} \times Z$ by one of the following sets:

$$
\mathscr{B}_{r}=\{(p, z) \in \mathscr{W} \times Z \mid \delta(p, z)<r\} \quad \text { for } \quad 0<r_{1}<r<r_{0} .
$$

We will now describe the singular set denoted by $\Sigma$ corresponding to the obstructions a), b). (We will see that obstruction c) occurs only for the global extension problem.) The set $\tilde{S}$ is a complex analytic set and has a stratification with strata consisting of smooth submanifolds. Since the basis is one dimensional, it is well known that there exist a stratification of $(\mathscr{W} \times \mathscr{U}, \widetilde{S})$ such that the rank of $d \pi$ is constant on each stratum and satisfying Thom $A_{\pi}$ condition. (See [Hi1].) (In the sequel we will always consider such a stratification.) We are indebted to C. Sabbah for numerous discussions and indications concerning the Propositions 4.3-4.6 and Lemma 4.4.

Definition 4.2. $\Sigma \subset \mathscr{U}$ is the projection on $\mathscr{U}$ of $\bar{M}$, for each stratum $M$ of $\tilde{S}$ in $\mathscr{W} \times \mathscr{U} \times Z$, such that $\left.d \pi\right|_{T M}=0$.

$\Sigma$ is analogous to the Landau variety in the study of Feynman integrals. We will call the points of $\Sigma$ Landau resonances. It is important to notice that the set $\Sigma$ in $\mathscr{U}$ depends on the set $\mathscr{W} \times Z$ used to make the contour deformations.

Proposition 4.3. $\Sigma$ is a finite set of points.

Proof. Since $\tilde{S}$ is an analytic set in $\mathscr{W} \times \mathscr{U} \times Z$ which extends outside $\mathscr{W} \times \mathscr{U} \times Z$, the number of strata of $\tilde{S}$ in $\overline{\mathscr{W}} \times \overline{\mathscr{U}} \times \bar{Z}$ is finite. Let $M$ be a stratum of $\tilde{S}$ such that $\left.d \pi\right|_{T M}=0$. By the curve selection lemma (see [Mi]), if $\left(p_{0}, \lambda_{0}, z_{0}\right) \in \bar{M}$, there exist a real analytic curve $\rho:\left[0, \varepsilon_{0}\left[\rightarrow \bar{M}\right.\right.$ such that $\rho(0)=\left(p_{0}, \lambda_{0}, z_{0}\right)$ and $\rho(t) \in M$, $\forall t>0$. Consider now the curve $\pi^{\circ} \rho(t)$ in $\mathscr{U}$. One has $d\left(\pi^{\circ} \rho(t)\right) \equiv 0$ since $\rho(t) \in M$, $\forall t>0$, so $\pi \circ \rho(t)=\pi\left(p_{0}, \lambda_{0}, z_{0}\right)=\lambda_{0}$. By the connectedness of $\bar{M}, \pi(\bar{M})=\lambda_{0}$, which proves the Proposition.

Let us now describe the set $\Sigma_{\infty}$ corresponding to obstruction c).

If $M$ is a stratum of $\tilde{S}$ in $\mathscr{W} \times \mathscr{U} \times Z, M_{\lambda}$ is a union of smooth submánifolds for $\lambda \in \mathscr{U}$. This is obvious if $\left.d \pi\right|_{T M} \neq 0$, and follows from the proof of Proposition 4.3, and Thom $A_{\pi}$ condition if $\left.d \pi\right|_{T M}=0$. For $\lambda \in \mathscr{U}$, we denote by $D(\lambda)$ the set of $r \in\left[r_{1}, r_{0}\right]$ such that for some stratum $M$ of $\tilde{S}, M_{\lambda}$ is tangent to $\partial B_{r}$. 
We have the following lemma:

\section{Lemma 4.4.}

i) $D(\lambda)$ is a finite subset of $\left[r_{1}, r_{0}\right]$.

ii) $K=\bigcup_{i \in \mathscr{U}}\{\lambda\} \times D(\lambda)$ is a closed set.

Proof.

i) can be proved as Proposition 4.4, using the critical variety $c M_{\lambda}$ of $M_{\lambda}$ with respect to the real analytic map $\delta . \overline{c \mathscr{M}}{ }_{\lambda}$ is a real analytic set and we can apply the real analytic version of the curve selection lemma to each connected component of $\overline{c \mathscr{M}}_{\lambda}$ to prove that $D(\lambda)$ is a finite set.

Let us now prove ii).

Let $\lambda_{0} \in \mathscr{U}, r_{0} \in\left[r_{1}, r_{0}\right]$, and assume that there exist a sequence $\left(\lambda_{n}, r_{n}\right) \rightarrow\left(\lambda_{0}, r_{0}\right)$, $\lambda_{n} \in \mathscr{U}, r_{n} \in D\left(\lambda_{n}\right)$. Then we can find a stratum $M$ of $\widetilde{S}$, a sequence $\left(p_{n}, z_{n}\right) \in M_{\lambda_{n}}$, such that $T_{\left(p_{n}, z_{n}\right)} M_{\lambda_{n}} \subset T_{\left(p_{n}, z_{n}\right)} \partial B_{r_{n}}$. By compactness we can assume that $\left(p_{n}, z_{n}\right)$ tends to some $\left(p_{0}, z_{0}\right) \in \partial B_{r_{0}}$, such that $\left(p_{0}, \lambda_{0}, z_{0}\right) \in \bar{M}$. If $\left(p_{0}, \lambda_{0}, z_{0}\right) \in M, T_{\left(p_{0}, z_{0}\right)} M_{\lambda_{0}} \subset$ $T_{\left(p_{0}, z_{0}\right)} \partial B_{r_{0}}$, and $r_{0} \in D\left(\lambda_{0}\right)$.

If $\left(p_{0}, \lambda_{0}, z_{0}\right) \in N$, for some stratum $N$ of $\tilde{S}$ adjacent to $M$, then using Thom $A_{\pi}$ condition, $T_{\left(p_{0}, z_{0}\right)} N_{\lambda_{0}} \subset T_{\left(p_{0}, z_{0}\right)} \partial B_{r_{0}}$, and $r_{0} \in D\left(\lambda_{0}\right)$, which proves that $K$ is closed.

From now on, we replace $\mathscr{W} \times Z$ by $B_{r_{0}}$, and consider $\partial B_{r_{0}}$ as the boundary of the holomorphy domain of $G(p, \lambda, z)$ in the variables $(p, z)$.

Definition 4.5. $\Sigma_{\infty}$ is the set of $\lambda \in \mathscr{U}$ such that $r_{0} \in D(\lambda)$

Proposition 4.6. It is possible to choose $r_{0}$ such that $\Sigma_{\infty}$ is disjoint from $\Sigma$ and is included in a closed subanalytic set of measure zero.

We refer to [Hi1] for the definition of subanalytic sets.

Proof. We first check that it is possible to choose $r_{0}$ such that $\Sigma_{\infty}$ is disjoint from $\Sigma$. From Proposition 4.5 ii), we can find some subinterval $\left[r_{1}^{\prime}, r_{0}^{\prime}\right]$ of $\left[r_{1}, r_{0}\right]$, and a small neighborhood $\mathscr{V}$ of $\Sigma$ in $\mathscr{U}$, such that $\left[r_{1}^{\prime}, r_{0}^{\prime}\right] \cap D(\lambda)=\varnothing, \forall \lambda \in \mathscr{V}$.

In the sequel the new $r_{0}$ will be chosen in $\left[r_{1}^{\prime}, r_{0}^{\prime}\right]$. We first take a semianalytic stratification $(M)$ of the pair $\left(\mathscr{U} \times \bar{B}_{r_{0}}, \widetilde{S}\right)$ (see [Hi1]) such that the strata of $(M)$ in $\mathscr{U} \times B_{r_{0}}$ are the strata of the stratification of $\left(\mathscr{U} \times B_{r_{0}}, \tilde{S}\right)$. Let us denote by $\hat{\Sigma}$ the union of the sets of critical values of the mappings $(p, \lambda, z) \in M \rightarrow(\lambda, \delta(p, z)) \in \mathscr{U} \times$ $\left[r_{1}, r_{0}\right]$, for all strata $M$ of $(M)$. It is easy to see that if $r \in D(\lambda),(\lambda, r) \in \hat{\Sigma}$. Moreover, $\hat{\Sigma}$ is a subanalytic set as a proper image of a semianalytic set in $\mathscr{U} \times \bar{B}_{r_{0}}$, and is of measure zero by Sard's theorem. By Fubini's theorem, we can find some $r_{3} \in\left[r_{1}^{\prime}, r_{0}^{\prime}\right]$ such that the fiber $\hat{\Sigma}_{r_{3}}$ in $\mathscr{U}$ of $\hat{\Sigma}$ over $r_{3}$ is of measure zero. This fiber is again a subanalytic set and contains the set $\Sigma_{\infty}$ if we take $r_{0}=r_{3}$. This proves the Proposition.

We have the following theorem, which is the main result of this section:

Theorem 4.7. Assume that $V$ belongs to $L_{\alpha}^{k}\left(C_{T}\right)$, for $k>m, \alpha>0$.

Then the following results hold:

i) Local Extension Problem: for any $\lambda_{0} \in \mathscr{U} \cap \mathbf{R}$, there exist a neighborhood $\mathscr{U}_{\lambda_{0}}$ of 
$\lambda_{0}$ in $\mathscr{U}$, such that $(H-\lambda)^{-1}$ extends holomorphically from $\{\operatorname{Im} \lambda>0\} \cap \mathscr{U}_{\lambda_{0}}$ to the universal covering $\left(\mathscr{U}_{\lambda_{0}} \backslash \Sigma\right)^{*}$, as a bounded operator from $L_{a}^{2}\left(\mathbf{R}^{m}\right)$ into $H_{-a}^{1}\left(\mathbf{R}^{m}\right)$ for $a>\alpha / 2$.

ii) Global Extension Problem: $(H-\lambda)^{-1}$ extends holomorphically from $\{\operatorname{Im} \lambda>0\} \cap \mathscr{U}$ to the universal covering $\left(\mathscr{U} \backslash \Sigma \cup \Sigma_{\infty}\right)^{*}$, as a bounded operator from $L_{a}^{2}\left(\mathbf{R}^{m}\right)$ into $H_{-a}^{1}\left(\mathbf{R}^{m}\right)$ for $a>\alpha / 2$.

Proof. Since $\alpha>0$, we can find $\mathscr{W}$ and $Z$ such that $\alpha>2\left(C(Z)+\sup |\operatorname{Im} p|_{p \in \mathscr{W}}\right)$, so that Proposition 4.1 applies to $\mathscr{W} \times \mathscr{U} \times Z$. We will follow the proof of Kobayashi [K]. Let us first prove ii). Let $\Delta_{i}^{ \pm}=\left\{(p, z) \in \Delta \mid p_{i}= \pm 1\right\}$ be the components of the boundary of $\Delta$, and let us denote by $\omega$ the operator valued form $G(p, \lambda, z) d p_{1} \wedge \cdots \wedge d p_{m-1} \wedge d z_{1} \wedge \cdots \wedge d z_{N}$.

Since $\omega$ is holomorphic of maximal degree, we have: $\left.d \omega\right|_{Y_{\lambda}}=0,\left.\omega\right|_{\tilde{F}_{\lambda}}=0$. This implies that if $\gamma$ is some chain in $Y_{\lambda}, \int_{\gamma} \omega$ depends only on the homology class $[\gamma]$ of $\gamma$ in the relative homology group $\stackrel{\gamma}{H-1}_{m}\left(Y_{\lambda}, \tilde{F}_{\lambda}\right)$.

We consider now the problem of extending $(H-\lambda)^{-1}=\int_{\Delta} \omega$ from $\operatorname{Im} \lambda>0$, to some point $\lambda_{1}$ along a path $\ell:[0,1] \rightarrow \mathscr{U}$, with $\operatorname{Im} \ell(0)>0, \ell(1)=\lambda_{1}$. Applying the result of Kobayashi ([K] Theorem 1.3), we get that $(H-\lambda)^{-1}$ can be extended holomorphically along $\ell$ if there exist a deformation $\gamma_{t}:\left(\Delta, \Delta_{i}^{ \pm}\right) \rightarrow\left(Y_{\ell(t)}, \widetilde{F}_{b, \ell(t)}^{ \pm}\right)$of $\Delta$ such that $\gamma_{0}=\operatorname{Id}$ and $\gamma:(t, s) \in[0,1] \times \Delta \rightarrow \gamma_{t}(s)$ is continuous. This reduces the problem of holomorphic continuation to a problem of finding a continuous deformation of the relative cycle $\Delta$ in $H_{m-1}\left(Y_{\lambda}, \widetilde{F}_{\lambda}\right)$ along the path $\ell$.

Let now $\ell:[0,1] \rightarrow \mathscr{U} \backslash \Sigma \cup \Sigma_{\infty}$ be a path with $\ell(0)=\lambda_{0}$, Im $\lambda_{0}>0$. Let $J$ be the set of $t \in[0,1]$ such that $\Delta$ can be deformed along $\ell$ between 0 and $t$ satisfying the above conditions. $J$ is obviously open and since $[0,1]$ is connected it suffices to prove that $J$ is closed to prove that $I=J$.

Let $t_{n} \in J$ a sequence with $t_{n} \rightarrow t_{0}$ when $n \rightarrow+\infty . \lambda_{1}=\ell\left(t_{0}\right)$ belongs to $\mathscr{U} \backslash \Sigma \cup \Sigma_{\infty}$, and if $V$ is a small neighborhood of $\lambda_{1}, M_{\lambda}$ is transversal to $\partial B_{r_{0}}$ for all $\lambda \in V$, all strata $M$ of $\tilde{S}$, if $r_{0}$ is defined in Proposition 4.6. Then we can apply to $\bar{B}_{r_{0}} \times V$ the local isotropy lemma of [F.K.]. This implies that $\pi: \bar{B}_{r_{0}} \times V \rightarrow V$ is a locally trivial fibration with respect to $S \cap\left(\bar{B}_{r_{0}} \times V\right), F_{i}^{ \pm} \cap\left(\bar{B}_{r_{0}} \times V\right)$.

This implies that one can find a deformation of $\gamma_{\ell\left(t_{n}\right)}$ along the part of $\ell$ which stays in $V$, such that $\gamma_{\ell(t)} \subset \bar{B}_{r} \backslash S_{\ell(t)}$ and the faces of $\gamma_{\ell(t)}$ are in the submanifolds $F_{i, \ell(t)}^{ \pm}$. This implies that $I=J$, hence that $(H-\lambda)^{-1}$ can be extended along any path in $\mathscr{U} \backslash \Sigma \cup \Sigma_{\infty}$.

Then it follows from the monodromy Theorem that $(H-\lambda)^{-1}$ extends as a function on $\left(\mathscr{U} \backslash \Sigma \cup \Sigma_{\infty}\right)^{*}$, which proves ii). Let us now prove i). From Lemma 4.5, we can find some $r_{0}>0$, some neighborhood $\mathscr{U}_{\lambda_{0}}$ of $\lambda_{0}$ in $\mathscr{U}$, such that $r_{0} \notin D(\lambda)$, $\forall \lambda \in \mathscr{U}_{\lambda_{0}}$. Then the result follows by applying the arguments above to $\bar{B}_{r_{0}} \times \mathscr{U}_{\lambda_{0}}$. This proves the Theorem.

We will now study the behavior of $(H-\lambda)^{-1}$ near a Landau resonance.

We first recall some definitions.

Definition 4.8. An operator-valued function $M(\lambda)$ is of finite determination near some point $\lambda_{0}$ if there exist a neighborhood $\mathscr{V}$ of $\lambda_{0}$ such that the branches of $M(\lambda)$ over any simply connected subset of $\mathscr{V} \backslash\left\{\lambda_{0}\right\}$ span a vector space of finite dimension in $\mathscr{L}\left(L_{a}^{2}\left(\mathbf{R}^{m}\right), H_{-a}^{1}\left(\mathbf{R}^{m}\right)\right)$. 
$M(\lambda)$ has a moderate growth at a point $\lambda_{0} \in \Sigma$, if there exist a neighborhood $V$ of $\lambda_{0}$ in $\mathscr{U}$ such that for any simply connected subset $\widetilde{V}$ of $V \backslash\left\{\lambda_{0}\right\}$, for any branch of $M(\lambda)$ on $\tilde{V}$, denoted by $\tilde{M}(\lambda)$, there exist $C_{0}, N_{0}>0$ such that:

$$
\|\tilde{M}(\lambda)\| \leqq C_{0}\left|\lambda-\lambda_{0}\right|^{-N_{0}} \text {. }
$$

Here $\|\tilde{M}(\lambda)\|$ is the operator norm in $\mathscr{L}\left(L_{a}^{2}\left(\mathbf{R}^{m}\right), H_{-a}^{1}\left(\mathbf{R}^{m}\right)\right)$.

-an analytic set $\tilde{S}$ in $\mathscr{W} \times \mathscr{U} \times Z$ is a divisor with normal crossings if near any point $\left(p_{0}, \lambda_{0}, z_{0}\right) \in \tilde{S}$, there exist holomorphic coordinates $\left(z_{1}, \ldots, z_{m+N}\right)$ such that $\widetilde{S}$ is given by the equation $z_{1} \ldots z_{k}=0$ near $\left(p_{0}, \lambda_{0}, z_{0}\right)$.

To study the growth of $(H-\lambda)^{-1}$ near a point of $\Sigma$, we need a geometric hypothesis on $\tilde{S}$.

We first add to $\tilde{S}$ the fiber $\pi^{-1}\left(\lambda_{0}\right)$ for each $\lambda_{0} \in \Sigma$, which does not change the set $\Sigma$.

Hironaka desingularisation theorem says that there exist an analytic space $X$ and a proper morphism $\beta: X \rightarrow \mathscr{W} \times \mathscr{U} \times Z$ such that:

$-\beta: X \backslash \beta^{-1}(\tilde{S}) \rightarrow \mathscr{W} \times \mathscr{U} \times Z \backslash \tilde{S}$ is an isomorphism.

$-\widetilde{S}^{\prime}=\beta^{-1}(\widetilde{S})$ is a divisor with normal crossings. (See [Hi2]).

We make the same hypothesis as in Mercier [Me]:

(T) There exist a stratification $(\tilde{M})$ of the pair $\left(X, \tilde{S}^{\prime}\right)$ such that for each $\tilde{M}$ there exist a stratum $M$ of $(\mathscr{W} \times \mathscr{U} \times Z, \tilde{S})$ such that $\left.\beta\right|_{\tilde{M}}: \tilde{M} \rightarrow M$ is a submersion.

The hypothesis is made to ensure that the strata of $\tilde{S}^{\prime}$ intersecting $\beta^{-1}\left(\partial B_{r_{0}} \times V\right)$ intersect it transversally.

We have the following theorem:

\section{Theorem 4.9.}

i) $(H-\lambda)^{-1}$ is of finite determination near any point of $\Sigma$.

ii) if condition $(T)$ holds, $(H-\lambda)^{-1}$ has a moderate growth near any point of $\Sigma$.

Proof. Since the properties of finite determination and moderate growth are local, we can consider $(H-\lambda)^{-1}$ near a point $\lambda_{1} \in \Sigma$. Let us consider a branch of $(H-\lambda)^{-1}$ near $\lambda_{1}$, obtained by analytic continuation along a homotopy class $[\gamma]$ in $\mathscr{U} \backslash \Sigma \cup \Sigma_{\infty}$. The continuation of $(H-\lambda)^{-1}$ in $\left(V \mid\left\{\lambda_{1}\right\}\right)^{*}$, where $V$ is a small neighborhood of $\lambda_{1}$, is obtained by deforming the integration cycle $\Delta$ in (4.2) inside the set $B_{r_{0}}$. We claim that it is possible to choose $r_{0}$ in Proposition 4.6 such that $r_{0} \notin D(\lambda), \forall \lambda \in V$ and the following condition holds:

$\left(T^{\prime}\right)$ any stratum of $\tilde{S}$ intersecting $\partial B_{r_{0}} \times \mathscr{U}$ intersect it transversally. Indeed it suffices to remark as in Lemma 4.5 that the set of critical values of $\delta$ on $M$ is finite, for all strata $M$ of $\tilde{S}$.

Let $\lambda_{0}$ a point in $V \backslash\left\{\lambda_{1}\right\}$. We will adapt the arguments of Mercier [Me] to our problem. Let us assume that one branch of $(H-\lambda)^{-1}$ at $\lambda_{0}$ is obtained by integrating in (4.2) on some relative cycle $\gamma_{0}$.

We introduce the locally finite family of analytic sets given by $S_{\lambda_{0}}, F_{i, \lambda_{0}}^{ \pm}$and $\partial B_{r_{0}}$. By Lojasiewicz Theorem (see [Me]), we can find a semianalytic triangulation of $\bar{B}_{r_{0}}$ which is finite and compatible with this family. This induces a triangulation of the pair $\left(\bar{B}_{r_{0}} \backslash S_{\lambda_{0}}, \widetilde{F}_{\lambda_{0}}\right)$ by $(K, L)$, where $K$ is the simplicial complex made of the simplexes of the previous triangulation which do not intersect $S_{\lambda_{0}}$ and $L$ is the simplicial complex made of the simplexes of the previous triangulation which intersect $\tilde{F}_{\lambda_{0}}$ and not $S_{\lambda_{0}}$. 
Then $H_{m-1}\left(\bar{B}_{r_{0}} \backslash S_{\lambda_{0}}, \widetilde{F}_{\lambda_{0}}\right)$ is isomorphic to $H_{m-1}(|K|,|L|)$ and we can write $\left[\gamma_{0}\right]$ using the simplexes of the triangulation of $(|K|,|L|):\left[\gamma_{0}\right]=\sum_{j \in J_{0}} b_{j} \sigma_{j}, b_{j} \in \mathbf{Z}, J_{0}$ a
finite set.

For $\lambda$ near $\lambda_{0}$, we can also take $\left[\gamma_{\lambda}\right]=\sum_{j \in J_{0}} b_{j} \sigma_{j}$.

Then $(H-\lambda)^{-1}=\sum_{j \in J_{0}} b_{j} \int_{\sigma_{j}} \omega$.

It is clear that $\omega=G(p, \lambda, z) d p_{1} \wedge \cdots \wedge d p_{m-1} \wedge d z_{1} \wedge \cdots \wedge d z_{N}$ is of finite determination on any of the simply connected subsets $\sigma_{j}$, since the multivaluedness of $\omega$ comes only from the $\left(\lambda-\left(n_{i}+p\right)^{2}\right)^{1 / 2}$, which are of finite determination.

So $(H-\lambda)^{-1}$ is a finite sum of the functions $\int_{\sigma_{j}} \omega$, each of finite determination, which proves i).

We prove now ii): we consider a point $\lambda_{0} \in \Sigma$.

Let us denote by $\beta: X \rightarrow \mathscr{W} \times \mathscr{U} \times Z$ the desingularisation of $\tilde{S}$ in $\mathscr{W} \times \mathscr{U} \times Z$. We can write $(H-\lambda)^{-1}=\int_{\gamma_{\lambda}} \omega=\int_{\beta_{*} \gamma_{\lambda}^{\prime}} \omega=\int_{\gamma_{\lambda}^{\prime}} \beta^{*} \omega$, where $\gamma_{\lambda}^{\prime}=\left(\beta^{-1}\right)_{*} \gamma_{\lambda}$. (Here $\gamma_{\lambda}^{\prime}$ exist because $\beta$ is an isomorphism outside $\tilde{S}$.) Using condition $(T)$, we see that $\beta^{-1}\left(\partial B_{r_{0}} \times \mathscr{U}\right)$ is transversal to all strata of $\widetilde{S}^{\prime}$. So denoting again $\widetilde{S}^{\prime}$ by $\tilde{S}$, we are reduced to the case where $\pi^{-1}\left(\lambda_{0}\right) \subset \widetilde{S}, \widetilde{S}$ is a divisor, and $\partial B_{r_{0}} \times \mathscr{U}$ is transversal to all strata of $\tilde{S}$.

We fix a small neighborhood $V$ of $\lambda_{0}$, in which we will estimate the growth of $(H-\lambda)^{-1}$.

We can now finish the proof as in [Me].

We will only indicate the principal steps of the proof. Modulo a change of coordinates, we can assume that $\lambda_{0}=0$. The idea of the proof is to lift the radial vector field on $\mathbf{C}: \zeta=-(\lambda(\partial / \partial \lambda)+\bar{\lambda}(\partial / \partial \bar{\lambda}))=-r(\partial / \partial r)$, where $r=|\lambda|$, to a vector field $\xi$ in $\mathscr{W} \times V$ which is tangent to all strata of $\tilde{S}$ and to $\partial B_{r_{0}} \times V$. This vector field is then used to construct the deformations of $\gamma_{\lambda}$ as $\lambda$ tends to 0 along an integral curve of $\zeta$, which is of the form: $\alpha_{s}\left(\lambda_{1}\right)=\lambda_{1} e^{-s}$ for $s \in \mathbf{R}^{+}$.

Step 1: Construction of $\xi$.

We will construct $\xi$ locally near any point of $B_{r_{0}} \times V$ and patch together the local vector fields with a partition of unity.

1. near $\left(p_{0}, \lambda_{0}, z_{0}\right) \in \bar{B}_{r_{0}} \times V \backslash\left(\tilde{S} \cup \partial B_{r_{0}} \times V\right)$ :

$$
\text { we take } \xi=\left(\lambda \frac{\partial}{\partial \lambda}+\bar{\lambda} \frac{\partial}{\partial \bar{\lambda}}\right) \text {. }
$$

2. $\operatorname{near}\left(p_{0}, \lambda_{0}, z_{0}\right) \in \bar{B}_{r_{0}} \times V \cap\left(\widetilde{S} \backslash \partial B_{r_{0}} \times V\right)$ :

Since $\tilde{S}$ is a divisor, we can take a coordinate chart near $\left(p_{0}, \lambda_{0}, z_{0}\right),\left(z_{1}, \ldots, z_{m+N}\right)$ such that $\tilde{S}$ is given near $\left(p_{0}, \lambda_{0}, z_{0}\right)$ by the equation $z_{1} \ldots z_{p}=0$, and $\left(p_{0}, \lambda_{0}, z_{0}\right)=$ $(0, \ldots, 0)$. Since $\pi^{-1}(0) \subset \widetilde{S}$, by the Nullstellensatz (see [Me] p. 82) we see that $\pi(z)=r_{0}(z) z_{1}^{\alpha_{1}} \cdots z_{k}^{\alpha_{k}}, \alpha_{1}, \ldots, \alpha_{k} \in \mathbf{N}, r_{0}(0) \neq 0, k \leqq p$. Changing for example $z_{1}$, we can assume that $\pi(z)=z_{1}^{\alpha_{1}} \cdots z_{k}^{\alpha_{k}}$. We can take

$$
\xi=-\frac{1}{\alpha_{1}}\left(z_{1} \frac{\partial}{\partial z_{1}}+\bar{z}_{1} \frac{\partial}{\partial z_{1}}\right)
$$

and we have $\pi_{*} \xi=\zeta$. 
3. $\operatorname{near}\left(p_{0}, \lambda_{0}, z_{0}\right) \in \bar{B}_{r_{0}} \times V \cap\left(\tilde{S} \cap \partial B_{r_{0}} \times V\right)$ :

As before we can find a coordinate chart such that $\tilde{S}$ has the equation $z_{1} \cdots z_{p}=0$, and $\pi(z)=z_{1}^{\alpha_{1}} \cdots z_{k}^{\alpha_{k}}$.

Since $\partial B_{r_{0}}$ is transversal to each stratum of $\tilde{S}$, we can extend the set of local coordinates $\left(\operatorname{Re} z_{1}, \operatorname{Im} z_{1}, \ldots, \operatorname{Re} z_{p}, \operatorname{Im} z_{p}\right)$ by $u_{p+1}, v_{p+1}, \ldots, u_{m+N}, v_{m+N}$ such that $u_{p+1}=0$ is a $C^{\infty}$ equation of $\partial B_{r}$ near $\left(p_{0}, \lambda_{0}, z_{0}\right)$. We take

$$
\xi=-\frac{1}{\alpha_{1}}\left(z_{1} \frac{\partial}{\partial z_{1}}+\bar{z}_{1} \frac{\partial}{\partial \bar{z}_{1}}\right)
$$

$\xi$ is tangent to $S$ and to $\partial B_{r_{0}}$, and $\pi_{*} \xi=\zeta$.

4. near $\left(p_{0}, \lambda_{0}, z_{0}\right) \in \bar{B}_{r_{0}} \times V \cap\left(\partial B_{r_{0}} \times U \backslash \tilde{S}\right)$ :

We can take

$$
\xi=-\left(\lambda \frac{\partial}{\partial \lambda}+\bar{\lambda} \frac{\partial}{\bar{\partial}}\right)
$$

$\xi$ is tangent to $\partial B_{r_{0}}$. We now patch together $\xi$ with a $C^{\infty}$ partition of unity in $\bar{B}_{r_{0}+\varepsilon} \times V_{\varepsilon}$, where $V_{\varepsilon}$ is a small neighborhood of $V$. We obtain a vector field supported in $\bar{B}_{r_{0}+\varepsilon} \times V_{\varepsilon}$.

Step 2: Estimates of $(H-\lambda)^{-1}$.

We want now to control the growth of $(H-\lambda)^{-1}=\int_{\gamma_{2}} \omega$, when $\lambda$ tends to 0 along a ray $\alpha_{s}\left(\lambda_{1}\right)=\lambda_{1} e^{-s}$.

Here for $\lambda \in V \backslash\left\{\lambda_{0}\right\}, \gamma_{\lambda}$ is a deformation of the cycle $\gamma_{\lambda_{1}}$ which stays in the ball $\bar{B}_{r_{0}}$. The integral curves of the vector field $\xi$ induce a 1-parameter family of diffeomorphisms $j_{s}=\exp (s \xi)$, from $\left(\bar{B}_{r_{0}} \backslash \tilde{S}_{\lambda_{1}}\right) \times\left\{\lambda_{1}\right\}$ into $\left(\bar{B}_{r_{0}} \backslash \tilde{S}_{\alpha_{s}\left(\lambda_{1}\right)}\right) \times\left\{\alpha_{s}\left(\lambda_{1}\right)\right\}$. We use here the fact that $\xi$ is tangent to $\partial B_{r_{0}}$ and $\tilde{S}$, and that $\pi_{*} \xi$ is the radial vector field pointing inwards. Moreover $j_{s}$ is a homeomorphism from the pair

$$
\left(\bar{B}_{r_{0}} \backslash S_{\lambda_{1}}, \widetilde{F}_{\lambda_{1}}\right) \text { to the pair }\left(\bar{B}_{r_{0}} \backslash \tilde{S}_{\alpha_{s}\left(\lambda_{1}\right)}, \tilde{F}_{\alpha_{s}\left(\lambda_{1}\right)}\right) \text {. }
$$

Since $H_{m-1}\left(\bar{B}_{r_{0}} \backslash S_{\lambda}, \widetilde{F}_{\lambda}\right)$ is a locally constant sheaf over $V$, (using for example the triangulation in the proof of i)), we see that $\left[\gamma_{\alpha_{s}\left(\lambda_{1}\right)}\right]=\left(j_{s}\right)_{*}\left[\gamma_{\lambda_{1}}\right]$.

So we can write:

$$
\left(H-\alpha_{s}\left(\lambda_{1}\right)\right)^{-1}=\int_{(j s)_{* \gamma_{\lambda_{1}}}} \omega=\int_{\gamma_{\lambda_{1}}} j_{s}^{*} \omega .
$$

To prove ii) it remains to show that $\left\|j_{s}^{*} \omega\right\| \leqq C e^{c s}$, where $\|\quad\|$ is the norm induced by the Riemannian structure on $\mathscr{W} \times Z$, uniformly on $\bar{B}_{r_{0}}$ and when $\lambda_{1}$ is on some arc of circle, $\left\{\theta_{0} \leqq \operatorname{Arg} \lambda_{1} \leqq \theta_{1},\left|\lambda_{1}\right|=\varepsilon_{0}\right\}$. This can be done as in the proof of [Me] Theorem 3.1, with the modifications of [Me] Theorem 3.2.

Behavior of the Resolvent Near a Landau Resonance in Generic Cases. We will now study more precisely the behavior of $(H-\lambda)^{-1}$ near some points of $\Sigma$. We consider the case of a Landau resonance $\lambda_{0} \in \Sigma$ generated by a pinch of the integration cycle at a point $\left(p_{0}, z_{0}\right) \in \bar{B}_{r_{0}}$. For simplicity, we will only consider Landau resonances created by polar singularities, i.e. by strata of $\tilde{S}$ away from $\lambda=\left(n_{i}+p\right)^{2} i=1, \ldots, N$. However, monodromy formulas for $(H-\lambda)^{-1}$ near a Landau resonance created by ramified singularities can be obtained using the results of Pham ([P] Chapter VII). 
We make the following hypotheses;

(4.5) there exist neighborhoods $V$ of $\lambda_{0}, \tilde{W}$ of $\left(p_{0}, z_{0}\right)$ such that if $\lambda \in V,(p, z) \in \bar{B}_{r_{0}} \backslash \tilde{W}$ then $(p, \lambda, z) \notin S$, and $\left(p_{0}, \lambda_{0}, z_{0}\right)$ is the only critical point of $\pi$ in $\tilde{W} \times V$.

$$
S_{\lambda_{0}} \cap F_{\lambda_{0}}=\varnothing \text {. }
$$

(4.7) near $\left(p_{0}, \lambda_{0}, z_{0}\right) S$ is the union of the complex hypersurfaces $S_{1}, \ldots, S_{k}$ intersecting in general position at $\left(p_{0}, \lambda_{0}, z_{0}\right)$. This means that near $\left(p_{0}, \lambda_{0}, z_{0}\right)$ $S_{i}$ has a irreducible equation $s_{i}(p, \lambda, z)=0$ with $s_{i}\left(p_{0}, \lambda_{0}, z_{0}\right)=0, d s_{1}, \ldots, d s_{k}$ linearly independent at $\left(p_{0}, \lambda_{0}, z_{0}\right)$. (4.8) $\left(p_{0}, \lambda_{0}, z_{0}\right)$ is a non-degenerate critical point of $\pi$ for the stratum $A=\bigcap_{i=1}^{k} S_{i}$
and is not a critical point of any other stratum of $S$.

(4.9) $S_{i}$ are polar manifolds of

$$
G(p, \lambda, z)=\frac{M(p, \lambda, z)}{f(p, \lambda, z)} \prod_{1}^{N}\left(\lambda-\left(n_{i}+p\right)^{2}\right)^{1 / 2}\left(z_{1}^{2}-\lambda+\left(n_{i}+p\right)^{2}\right)^{-1} .
$$

We note that it is possible to choose $M(p, \lambda, z)$ and $f(p, \lambda, z)$ near $\left(p_{0}, \lambda_{0}, z_{0}\right)$ such that $M\left(p_{0}, \lambda_{0}, z_{0}\right)$ is a finite rank operator. This follows easily from the holomorphic Fredholm theorem.

We consider the behavior of one branch of $(H-\lambda)^{-1}$, denoted by $(H-\lambda)_{\gamma}^{-1}$ obtained by integrating $G(p, \lambda, z)$ on a cycle $\gamma$, for $\lambda$ near $\lambda_{0}, \lambda \neq \lambda_{0}$.

Using (4.5), we can write $\gamma=\tilde{\gamma}+\sigma$, where $\sigma$ is an absolute cycle in $\tilde{W}$ and $\tilde{\gamma}$ avoids $S_{\lambda_{0}}$.

So $\int_{\tilde{\tilde{J}}} G(p, \lambda, z) d p_{1} \wedge \cdots \wedge d p_{m-1} \wedge d z_{1} \cdots \wedge d z_{N}$ is holomorphic for $\lambda$ near $\lambda_{0}$, and modulo a holomorphic term, we can assume that $\gamma=\sigma$ is an absolute cycle. We will denote by $N$ the intersection index of $\sigma$ with the vanishing cell $e$ defined by the manifolds $S_{1}, \ldots, S_{k}$. (See [P] Chap. V for the definitions of $N$ and $e$.) Practically $N$ is zero when the path $\sigma$ is not pinched between the $S_{1}, \ldots, S_{k}$.

An interesting question is whether the set of potentials in $L_{\alpha}^{\infty}\left(C_{T}\right)$ such that $S$ has the above structure near any critical point in $W \times \mathscr{U} \times Z$ is an open dense set of $L_{\alpha}^{\infty}\left(C_{T}\right)$.

Under the hypotheses above, we can write near $\left(p_{0}, \lambda_{0}, z_{0}\right), G(p, \lambda, z)$ as:

$$
\frac{\tilde{M}(p, \lambda, z)}{s_{1}^{\alpha_{1}}(p, \lambda, z) \cdots s_{k}^{\alpha_{k}}(p, \lambda, z)} \text { for } \alpha_{1}, \ldots, \alpha_{k} \in \mathbf{N}, \tilde{M} \text { as } M .
$$

The case when some $\alpha_{i}$ are bigger than 1 means that there is a constant degeneracy in the singularities of $\left(1+V K_{0}(p, \lambda, z)\right)^{-1}$. This happens for example if the crystalline material represented by the potential $V$ has a symmetry group. We put then $\alpha=\alpha_{1}+\cdots+\alpha_{k}$.

We have the following theorem:

Theorem 4.10. Under the hypotheses (4.i) $i=5, \ldots, 9$, the branch $(H-\lambda)_{\gamma}^{-1}$ can be written for $\lambda$ near $\lambda_{0}$ as:

i) if $m+N+k$ is odd:

$$
(H-\lambda)_{\gamma}^{-1}=E_{0}(\lambda)+C_{0} N\left(\lambda-\lambda_{0}\right)^{(m+N+k) / 2-1-\alpha}\left(M\left(p_{0}, \lambda_{0}, z_{0}\right)+\left(\lambda-\lambda_{0}\right) E_{1}(\lambda)\right),
$$


ii) if $m+N+k$ is even, $m+N+k \geqq 2 \alpha+2$ :

$(H-\lambda)_{\gamma}^{-1}=E_{0}(\lambda)+C_{0} N\left(\lambda-\lambda_{0}\right)^{(m+N+k) / 2-1-\alpha} \log \left(\lambda-\lambda_{0}\right)\left(M\left(p_{0}, \lambda_{0}, z_{0}\right)+\left(\lambda-\lambda_{0}\right) E_{1}(\lambda)\right)$,

iii) if $m+N+k$ is even, $m+N+k<2 \alpha+2$ :

$$
\begin{aligned}
(H-\lambda)_{\gamma}^{-1}= & E_{0}(\lambda)+C_{0} N\left(\lambda-\lambda_{0}\right)^{(m+N+k) / 2-1-\alpha}\left(M\left(p_{0}, \lambda_{0}, z_{0}\right)+\left(\lambda-\lambda_{0}\right) E_{1}(\lambda)\right) \\
& +N \log \left(\lambda-\lambda_{0}\right) \times E_{2}(\lambda),
\end{aligned}
$$

iv) if $m+N=k$ :

$$
(H-\lambda)_{\gamma}^{-1}=E_{0}(\lambda)+C_{0} N\left(\lambda-\lambda_{0}\right)^{m+N-\alpha-1}\left(M\left(p_{0}, \lambda_{0}, z_{0}\right)+\left(\lambda-\lambda_{0}\right) E_{1}(\lambda)\right),
$$

where: $E_{0}, E_{1}, E_{2}$ are holomorphic functions in $\mathscr{L}\left(L_{a}^{2}\left(\mathbf{R}^{m}\right), H_{-a}^{1}\left(\mathbf{R}^{m}\right)\right)$, and $C_{0}$ is a non-vanishing constant.

Proof. We use the results of Leray [L] and Pham [P] as stated in the book of Pham (see [P] Chap. VI).

We first reduce ourselves to a case when $\tilde{M}(p, \lambda, z)$ is independent of $\lambda$. To do this, we use the fact (see [P] Sect. V.2) that under the hypotheses (4.7), (4.8), there exist a neighborhood of $\left(p_{0}, \lambda_{0}, z_{0}\right)$ still denoted by $\tilde{W} \times V$ and a holomorphic change of coordinates defined on $\tilde{W} \times V:(p, \lambda, z) \rightarrow(\tilde{p}(p, z, \lambda), \lambda)$ such that in the new coordinates $(\tilde{p}, \lambda)$ the functions $s_{1}, \ldots, s_{k}$ take the simple form:

$$
\begin{aligned}
& s_{1}=\lambda-\left(\tilde{p}_{1}+\cdots+\tilde{p}_{k-1}+\tilde{p}_{k}^{2}+\cdots+\tilde{p}_{m+N-1}^{2}\right)=\lambda-f(\tilde{p}) \\
& s_{2}=\tilde{p}_{1} \\
& \quad \vdots \\
& s_{k}=\tilde{p}_{k-1} .
\end{aligned}
$$

For simplicity, we will still denote by $(p, \lambda, z)$ the new coordinates. We can also assume that the cycle $\sigma$ is contained in $\widetilde{W}$, by subtracting to $(H-\lambda)^{-1}$ some operator holomorphic in $\lambda$. This can be done as in the proof of VI.2.1. in [P].

Using Taylor's formula, we can write:

$$
\begin{aligned}
\frac{\tilde{M}(p, \lambda, z)}{s_{1}^{\alpha_{1}} s_{2}^{\alpha_{2}} \cdots s_{k}^{\alpha_{k}}}= & \left.\sum_{0 \leqq \beta \leqq \alpha_{1}-1} \frac{1}{\beta !} \partial_{\lambda}^{\beta} \tilde{M}(p, \lambda, z)\right|_{\lambda=f(p, z)} s_{1}^{-\alpha_{1}+\beta} s_{2}^{-\alpha_{2}} \cdots s_{k}^{-\alpha_{k}} \\
& +\tilde{R}(p, \lambda, z) s_{2}^{-\alpha_{2}} \cdots s_{k}^{-\alpha_{k}}=\sum_{0 \leqq \beta \leqq \alpha_{1}-1} \tilde{M}_{\beta}(p, z) s_{1}^{-\alpha_{1}+\beta} s_{2}^{-\alpha_{2}} \cdots s_{k}^{-\alpha_{k}} \\
& +\tilde{R}(p, \lambda, z) s_{2}^{-\alpha_{2}} \cdots s_{k}^{-\alpha_{k}}
\end{aligned}
$$

From (4.8), it follows that $\left(p_{0}, \lambda_{0}, z_{0}\right)$ is not a critical point of $\pi$ for the stratum $S_{2} \cap \cdots \cap S_{k}$, hence $\int_{\sigma} \tilde{R}(p, \lambda, z) s_{2}^{-\alpha_{2}} \cdots s_{k}^{-\alpha_{k}} d p_{1} \wedge \cdots \wedge d p_{m-1} \wedge d z_{1} \wedge \cdots \wedge d z_{N}$ is holomorphic near $\lambda_{0}$.

So we are reduced to the study of $\int_{\sigma} \tilde{M}_{\beta}(p, z) s_{1}^{-\alpha_{1}+\beta} s_{2}^{-\alpha_{2}} \cdots s_{k}^{-\alpha_{k}} d p_{1} \wedge \cdots \wedge$ $d p_{m-1} \wedge d z_{1} \wedge \cdots \wedge d z_{N}$ for $\beta \leqq \alpha_{1}-1$.

Then the theorem follows directly by applying VI.2.1 in $[\mathrm{P}]$ to each of the terms in (4.10). In (4.10) only the first term corresponding to $\tilde{M}(p, f(p, z) z)$ contributes to the leading singularity at $\lambda=\lambda_{0}$. The only thing that we have to 
check is that some constant appearing in the formulas VI.2.1. of [P] is non-zero:

More precisely we can write:

$$
d \lambda=\sum_{i=1}^{k} a_{i} d s_{i} \quad \text { at } \quad\left(p_{0}, \lambda_{0}, z_{0}\right)
$$

Let us check that $a_{i} \neq 0$ for $i=1, \ldots, k$. If for example $a_{1}=0$, then $\left(p_{0}, \lambda_{0}, z_{0}\right)$ would be an element of the critical manifold of the stratum $S_{2} \cap \cdots \cap S_{k}$, which is excluded by (4.8). This concludes the proof of the theorem.

Remark 4.11. The interest of Theorem 4.10 is that $M\left(p_{0}, \lambda_{0}, z_{0}\right)$ is always a finite rank operator, but $M\left(p_{0}, \lambda_{0}, z_{0}\right)$ can be equal to zero in some cases. When $M\left(p_{0}, \lambda_{0}, z_{0}\right) \neq 0$, Theorem 4.10 shows that the leading singularity of $(H-\lambda)^{-1}$ at a Landau resonance $\lambda_{0}$ is of finite rank. Note however that $E_{1}(\lambda), E_{2}(\lambda)$ are in general not of finite rank.

We will now apply Theorem 4.10 in some simple cases where it is possible to compute explicitly $M\left(p_{0}, \lambda_{0}, z_{0}\right)$.

Landau Resonance Generated by a Simple Resonance Curve. We assume that $\left\{s_{1}(p, \lambda, z)=0\right\}$ is a component of $\{f(p, \lambda, z)=0\}$ and that (modulo a change of indices) $s_{i}(p, \lambda, z)=z_{i}^{2}-\lambda+\left(n_{i}+p\right)^{2}$ for $i=2, \ldots, k$. We denote by $z^{\prime \prime}$ the rest of the $z$ variables. Since we have assumed that $\left(z_{0}, p_{0}, \lambda_{0}\right)$ is away from the hypersurfaces $\lambda=\left(n_{i}+p\right)^{2}$, we can find determinations of the functions $\left(\lambda-\left(n_{i}+p\right)^{2}\right)^{1 / 2}$ near $\left(\lambda_{0}, p_{0}\right)$ such that $z_{i 0}=\left(\lambda_{0}-\left(n_{i}+p_{0}\right)^{2}\right)^{1 / 2}$, for $i=2, \ldots, k$. Then it is easy to check that $\left(p_{0}, \lambda_{0}, z_{0}\right)$ is a critical point of $\pi$ for $A$ if and only if $\left(p_{0}, \lambda_{0}, z_{0}^{\prime \prime}\right)$ is a critical point of $\pi$ for the hypersurface given by $s_{1}\left(p, \lambda,\left(\lambda-\left(n_{2}-p\right)^{2}\right)^{1 / 2}, \ldots,\left(\lambda-\left(n_{k}+p\right)^{2}\right)^{1 / 2}, z^{\prime \prime}\right)=0$. If we denote by $s_{1}\left(p, \lambda, z^{\prime \prime}\right)$ this new function, we have $\left(\partial s_{i} / \partial \lambda\right)\left(p_{0}, \lambda_{0}, z_{0}^{\prime \prime}\right) \neq 0$, i.e. $\lambda=\lambda_{0}$ is a simple root of $s_{1}\left(p_{0}, \lambda, z_{0}^{\prime \prime}\right)$.

For a function $u \in L_{\text {loc }}^{2}\left(\mathbf{R}^{m}\right)$, which is $p_{0}$-Floquet periodic (i.e. $e^{i\left\langle p_{0}, x\right\rangle} u$ is $T$-periodic), we denote by $R_{0}(\lambda)$ the following operator:

$$
R_{0}(\lambda) u=\sum_{n_{j} \in J}\left(\lambda-z_{j_{0}}^{2}-\left(n_{j}+p_{0}\right)^{2}\right) e^{-i\left\langle n_{j}+p_{0}, x\right\rangle} u_{n_{j}}(y),
$$

where $u_{n_{j}}(y)$ is the Fourier coefficient of order $n_{j}$ (in the $x$ variable) of $e^{i\left\langle p_{0}, x\right\rangle} u$. (In particular if $k=N+1, R_{0}\left(\lambda_{0}\right)=0$.) We will assume for simplicity that $m \geqq 3$. Then we have the following corollary:

Corollary 4.12. Under the hypotheses above, $M\left(p_{0}, \lambda_{0}, z_{0}\right)$ is a finite rank operator $\pi_{0}$ : satisfying: $\left(H-\lambda_{0}+R_{0}\left(\lambda_{0}\right)\right) \pi_{0}=\pi_{0}\left(H-\lambda_{0}+R_{0}\left(\lambda_{0}\right)\right)=0$.

Proof. We can write for $\lambda$ near $\lambda_{0}: \tilde{D}\left(p_{0}, \lambda, z_{0}\right) / f\left(p_{0}, \lambda, z_{0}\right)=\widetilde{E}_{0}(\lambda)+\tilde{\pi}_{0} / \lambda-\lambda_{0}$, where $\widetilde{E}_{0}(\lambda)$ is a holomorphic operator.

In the Appendix Proposition A.1, we prove that: $\left(H_{p_{0}}-\lambda_{0}+\tilde{R}_{0}\left(\lambda_{0}\right)\right) \tilde{\pi}_{0}=$ $\tilde{\pi}_{0}\left(H_{p_{0}}-\lambda_{0}+\tilde{R}_{0}\left(\lambda_{0}\right)\right)=0$, where $\widetilde{R}_{0}(\lambda)=e^{i\left\langle p_{0}, x\right\rangle} R_{0}(\lambda) e^{-i\left\langle p_{0}, x\right\rangle}$.

We have $G\left(p_{0}, \lambda, z_{0}\right)=E_{0}(\lambda)+\pi_{0} / \lambda-\lambda_{0}$, where $E_{0}(\lambda)$ is holomorphic and $\pi_{0} u=c(m, T) e^{-i\left\langle p_{0}, x\right\rangle} \tilde{\pi}$ for $u \in L_{a}^{2}\left(\mathbf{R}^{m}\right)$. From this we get easily that $\pi_{0}$ satisfies the corollary.

In paricular if $k=N+1$, the singular part of $(H-\lambda)^{-1}$ at $\lambda_{0}$ is associated with $p_{0}$-Floquet periodic solutions of $H u-\lambda_{0} u=0$. 


\section{Resonances Created by Impurities}

As discussed in the introduction, one of the main problems in atom-surface scattering is to investigate what are the effects introduced in scattering quantities by the presence of impurities. (See [Ge] Sect. II.C). We will consider the periodic potentials as a background potential and treat the impurities as a relatively compact perturbation.

Let us assume that the effect of impurities can be described with an additional real potential $W(x, y)$ with $W \in L_{\alpha}^{\infty}\left(\mathbf{R}^{m}\right)=\left\{V \in L^{\infty}\left(\mathbf{R}^{m}\right) \mid e^{\alpha(\langle x\rangle+\langle y\rangle)} V \in L^{\infty}\left(\mathbf{R}^{m}\right)\right\}$. As in Sects. III, IV, we can allow local singularities of $W$. (See Remark 5.2.)

We denote by $\widetilde{H}$ the Hamiltonian $\widetilde{H}=H+W$ with domain $H^{2}\left(\mathbf{R}^{m}\right)$. Then we have the following theorem.

Theorem 5.1. Assume that $V$ belongs to $L_{\alpha}^{k}\left(C_{T}\right)$ for $k>m, \alpha>0$ and $W \in L_{\alpha^{\prime}}^{\infty}\left(\mathbf{R}^{m}\right)$ for $\alpha^{\prime}>0$. Then the following results hold:

i) Local Extension Problem: for any $\lambda_{0} \in \mathscr{U} \cap \mathbf{R}$, there exist a neighborhood $\mathscr{U}_{\lambda_{0}}$ of $\lambda_{0}$ in $\mathscr{U}$ such that $(\tilde{H}-\lambda)^{-1}$ extends meromorphically from $\{\operatorname{Im} \lambda>0\} \cap \mathscr{U}_{\lambda_{0}}$ to $\left(\mathscr{U}_{\lambda_{0}} \backslash \Sigma\right)^{*}$ as a bounded operator from $L_{a}^{2}\left(\mathbf{R}^{m}\right)$ into $H_{-a}^{1}\left(\mathbf{R}^{m}\right)$ for $a>\frac{1}{2}\left(\alpha+\alpha^{\prime}\right)$, with poles in $\left(\mathscr{U}_{\lambda_{0}} \backslash \Sigma\right)^{*}$ having finite rank residues.

ii) Global Extension Problem: $(\tilde{H}-\lambda)^{-1}$ extends meromorphically from $\{\operatorname{Im} \lambda>0\}$ to $\left(\mathscr{U} \backslash \Sigma \cup \Sigma_{\infty}\right)^{*}$ as a bounded operator from $L_{a}^{2}\left(\mathbf{R}^{m}\right)$ into $H_{-a}^{1}\left(\mathbf{R}^{m}\right)$ for $a>\frac{1}{2}\left(\alpha+\alpha^{\prime}\right)$, with poles in $\left(\mathscr{U} \backslash \Sigma \cup \Sigma_{\infty}\right)^{*}$ having finite rank residues.

We will call resonances of $\tilde{H}$ the poles of $(\tilde{H}-\lambda)^{-1}$ in $\left(\mathscr{U}_{\lambda_{0}} \backslash \Sigma\right)^{*}$ or $\left(\mathscr{U} \backslash \Sigma \cup \Sigma_{\infty}\right)^{*}$.

Proof. As in Sect. III, we use the second resolvent formula: For $\operatorname{Im} \lambda>0$, we have:

$$
(\tilde{H}-\lambda)^{-1}=(H-\lambda)^{-1}\left(\mathbf{1}+W(H-\lambda)^{-1}\right)^{-1}=(H-\lambda)^{-1}(\mathbf{1}+\tilde{K}(\lambda))^{-1} .
$$

From Theorem 4.7 and the fact that $W \in L_{\alpha}^{\infty}\left(\mathbf{R}^{m}\right)$ for $\alpha>0$, we see that $\tilde{K}(\lambda)$ extends holomorphically from $\operatorname{Im} \lambda>0$ to $\left(\mathscr{U} \backslash \Sigma \cup \Sigma_{\infty}\right)^{*}$ as a compact operator on $L_{a}^{2}\left(\mathbf{R}^{m}\right)$. Since $\left(\mathscr{U} \backslash \Sigma \cup \Sigma_{\infty}\right)^{*}$ is connected and $1+\widetilde{K}(\lambda)$ is invertible for $\operatorname{Im} \lambda \gg 1$, the theorem follows from the analytic Fredholm theorem.

Remark 5.2. As in Sect. III, Theorem 5.1 still holds if

$$
e^{\alpha(\langle x\rangle+\langle y\rangle)} W(\Delta+1)^{-1 / 2} \text { is compact for } \alpha>0 .
$$

Let us make some comments on this result:

The resonances of $\tilde{H}$ live on the universal covering $\left(\mathscr{U} \backslash \Sigma \cup \Sigma_{\infty}\right)^{*}$ which means that one can discover new resonances by turning around the Landau resonances of $H$.

The physical interpretation of resonances in "upper sheets" of $\left(\mathscr{U} \backslash \Sigma \cup \Sigma_{\infty}\right)^{*}$ is not clear to us.

We have the following corollary:

Corollary 5.2. Let us denote by $\Gamma_{\mathbf{R}}$ the set of resonances of $\tilde{H}$ in $\left(\mathscr{U} \backslash \Sigma \cup \Sigma_{\infty}\right)^{*} \cap$ $\{\operatorname{Im} \lambda \geqq 0\}$, and by $\Sigma_{\mathbf{R}}$ the set $\Sigma \cap \mathbf{R}$. Then:

i) $\sigma_{\mathrm{pp}}(\tilde{H}) \subset \Gamma_{\mathbf{R}} \cup \Sigma_{\mathbf{R}}, \Gamma_{\mathbf{R}} \subset \sigma_{\mathrm{pp}}(\tilde{H})$,

ii) $\sigma_{\mathrm{sc}}(\tilde{H})$ is empty and the eigenvalues of $\tilde{H}$ can accumulate only at $\Sigma_{\mathbf{R}}$.

Proof. i) Can be proved easily by using an idea of Balslev-Combes [B.C]. We 
have the following formula, if $d \widetilde{E}_{\lambda}$ denotes the spectral measure of $\tilde{H}$ :

$$
\tilde{E}_{]-\infty, \lambda]}-\tilde{E}_{]-\infty, \lambda[}=s \lim _{z \rightarrow \lambda, \operatorname{Im} z>0}(z-\lambda)(\tilde{H}-z)^{-1} .
$$

Let $\lambda_{0} \in \sigma_{\mathrm{pp}}(\tilde{H})$. Then $\tilde{E}_{\left.]-\infty, \lambda_{0}\right]}-\tilde{E}_{]-\infty, \lambda_{0}[}=\tilde{E}_{\left\{\lambda_{0}\right\}} \neq 0$. Since $L_{a}^{2}\left(\mathbf{R}^{m}\right)$ is dense in $L^{2}\left(\mathbf{R}^{m}\right)$, we can find some $\varphi \in L_{a}^{2}\left(\mathbf{R}^{m}\right)$, such that $\left(\widetilde{E}_{\left\{\lambda_{0}\right\}} \varphi, \varphi\right)=\lim _{z \rightarrow \lambda_{0}, \operatorname{Im}_{z>0}}\left(z-\lambda_{0}\right)$ $\left((\tilde{H}-z)^{-1} \varphi, \varphi\right) \neq 0$. Then $(\tilde{H}-z)^{-1}$ must have a singularity at $z=\lambda_{0}$, so $\lambda_{0} \in \Gamma_{\mathbf{R}} \cup \Sigma_{\mathbf{R}}$. Suppose now that $\lambda_{0} \in \Gamma_{\mathbf{R}}$, and that $\lambda_{0} \notin \sigma_{\mathrm{pp}}(\tilde{H})$. Then for any $\varphi_{1}, \varphi_{2} \in L_{a}^{2}\left(\mathbf{R}^{m}\right)$, we have $\lim _{z \rightarrow \lambda_{0}, \operatorname{Im} z>0}\left(z-\lambda_{0}\right)\left((\tilde{H}-z)^{-1} \varphi_{1}, \varphi_{2}\right)=0$, which is impossible if we choose $\varphi_{1}$ and $\varphi_{2}$ correctly with respect to the residue in the Laurent expansion of $(\tilde{H}-z)^{-1}$ at $\lambda_{0}$. This proves i). To prove ii), we notice that Theorem 5.1 implies that $\sigma_{\text {sc }}(\tilde{H}) \subset \Gamma_{\mathbf{R}} \cup \Sigma_{\mathbf{R}}$ (see [Re-Si]). $\Gamma_{\mathbf{R}} \cup \Sigma_{\mathbf{R}}$ is a set of points having only a locally finite set of accumulation points, so it cannot support a continuous measure, which proves that $\sigma_{\mathrm{sc}}(\tilde{H})=\varnothing$. The properties of the eigenvalues of $(\tilde{H})$ follows directly from i).

Concerning the problem mentioned at the beginning of the section, we see that the effect of impurities is to add poles to the Landau resonances already present for a perfect crystalline surface. An obvious example where an impurity can create a pole of $(\tilde{H}-\lambda)^{-1}$ is by taking $W \leqq 0$ sufficiently negative to create a bound state of $\tilde{H}$,i.e. a real pole of $(\tilde{H}-\lambda)^{-1}$. According to Theorem 4.10 , the Landau resonances are typically branch points of $(H-\lambda)^{-1}$ (the case of a polar singularity is very unusual), and should be still singularities of $(\tilde{H}-\lambda)^{-1}$. (See the discussion below.) So the presence of impurities should be seen on scattering cross-sections or spectral functions since polar singularities give rise to the well-known Breit-Wigner shapes and singularities like $\left(\lambda-\lambda_{0}\right)^{-1 / 2}$ or $\log \left(\lambda-\lambda_{0}\right)$ produce very different resonance shapes.

We will now briefly discuss the singularity of $(\tilde{H}-\lambda)^{-1}$ near a Landau resonance $\lambda_{0}$, assuming that the hypotheses (4.5),..,(4.9) of Theorem 4.10 hold. Recall that it follows from this theorem that the Riemann surface on which $(H-\lambda)^{-1}$ is holomorphic and univalued for $\lambda$ near $\lambda_{0}$ is the Riemann surface $T$ of $\left(\lambda-\lambda_{0}\right)^{1 / 2}$ if $m+N+k$ is odd and of $\log \left(\lambda-\lambda_{0}\right)$ if $m+N+k$ is even. Then we have the following result:

Theorem 5.3. Under the hypotheses of Theorem 4.10, if $m+N+k>2 \alpha$ and $m+N+k \geqq 2 \alpha+2$ if $m+N+k$ is even, the number of resonances of $\tilde{H}$ on a sheet of $T$ in a small neighborhood of $\lambda_{0}$ is finite.

Proof. From (5.1) and Theorem 4.10, we have:

$$
\mathbf{1}+W(H-\lambda)^{-1}=\mathbf{1}+E_{0}(\lambda)+d(\lambda)\left(K_{1}+\left(\lambda-\lambda_{0}\right) E_{1}(\lambda)\right),
$$

where $E_{0}(\lambda), E_{1}(\lambda)$ are holomorphic and compact operators on $L_{a}^{2}\left(\mathbf{R}^{m}\right), K_{1}$ is a finite rank operator and:

$$
\begin{aligned}
& d(\lambda)=\left(\lambda-\lambda_{0}\right)^{(m+N+k) / 2-\alpha-1} \text { if } m+N+k \text { is odd } \\
& d(\lambda)=\left(\lambda-\lambda_{0}\right)^{(m+N+k) / 2-\alpha-1} \log \left(\lambda-\lambda_{0}\right) \quad \text { is } m+N+k \text { is even. }
\end{aligned}
$$

Since $m+N+k>2 \alpha, d(\lambda)\left(\lambda-\lambda_{0}\right)=0\left(\left(\lambda-\lambda_{0}\right)^{\varepsilon}\right)$ for some $\varepsilon>0$. 
Assume first that $m+N+k$ is odd. The if we introduce the variable $\left(\lambda-\lambda_{0}\right)^{1 / 2}$, i.e. we uniformize the Riemann surface $T$, the theorem follows directly from the meromorphic Fredholm theorem.

Assume now that $m+N+k$ is even. Without loss of generality we can put $\lambda_{0}=0$. Using the fact that $E_{0}(\lambda)$ is compact and holomorphic in $\lambda$, we can write:

$$
E_{0}(\lambda)=E_{0}(0)+R(\lambda)=K_{0}+R_{0}+R(\lambda), \quad \text { with }\|R(\lambda)\|=0\left(\left|\lambda-\lambda_{0}\right|\right), \quad\left|R_{0}\right| \leqq 1 / 2,
$$

$K_{0}$ of finite rank. If we introduce the dummy variable $t=\lambda d(\lambda)$, we can invert $1+R_{0}+R(\lambda)+t E_{1}(\lambda)$ by a Neumann series for $|\lambda| \leqq \varepsilon_{0},|t| \leqq \varepsilon_{0} \cdot\left(1+R_{0}+R(\lambda)+\right.$ $\left.t E_{1}(\lambda)\right)^{-1}=1+F(t, \lambda)$, where $F(t, \lambda)$ is holomorphic for $|\lambda| \leqq \varepsilon_{0},|t| \leqq \varepsilon_{0}$.

Using again the second resolvent formula, we see that we are reduced to the inversion of $1+K_{0}(t, \lambda)+d(\lambda) K_{1}(t, \lambda)$ for $t=\lambda d(\lambda)$, where $K_{i}(t, \lambda)=K_{i}(1+F(t, \lambda))$.

As in the proof of Theorem 3.5, we are reduced to the inversion of a finite dimensional matrix $1+K_{0}(t, \lambda)+d(\lambda) K_{1}(t, \lambda)=1 / \lambda\left(\lambda \mathbf{1}+\lambda K_{0}(t, \lambda)+t K_{1}(t, \lambda)\right)=$ $(1 / \lambda) M(t, \lambda)$.

So $\lambda$ with $0<|\lambda| \leqq \varepsilon_{0}$ is a resonance of $\tilde{H}$ if and only if $f(\lambda d(\lambda), \lambda)=0$, where $f(t, \lambda)=\operatorname{det}(M(t, \lambda))$.

Using Taylor's formula in $\lambda$, we can write:

$$
f(t, \lambda)=\sum_{n=N}^{\infty} a_{n}(t) \lambda^{n}=\lambda^{N} g(t, \lambda), \quad \text { with } \quad a_{N}(t) \neq 0 .
$$

(We use here the fact that $g(t, \lambda) \neq 0$, since the resonances of $\tilde{H}$ are discrete in $\left\{\lambda|0<| \lambda \mid \leqq \varepsilon_{0}\right\}$. We can replace $f(t, \lambda)$ by $g(t, \lambda)$, and since $a_{N}(t) \not \equiv 0$, there exist $N_{0} \in \mathbf{N}$ such that $\partial_{t}^{N_{0}} g(0,0) \neq 0$. Using Weierstrass preparation theorem, we can hence replace $g(t, \lambda)$ by a polynomial in $t$ of degree $N_{0}$ :

$$
g(t, \lambda)=t^{N_{0}}+b_{1}(\lambda) t^{N_{0}-1}+\cdots+b_{N_{0}}(\lambda) .
$$

By Puiseux theorem, the solutions of $g(t, \lambda)=0$ are branches of analytic functions $t_{i}(\lambda), i=1, \ldots, M_{0}$, with:

$$
t_{i}(\lambda)=\lambda^{\alpha_{i}}\left(c_{i}+O\left(\lambda^{\varepsilon}\right)\right), \quad \alpha_{i} \in \mathbf{Q}, \quad \varepsilon>0, \quad \text { if } \quad t_{i}(0)=0 .
$$

It is then easy to check that the number of solutions of $\lambda d(\lambda)=t_{i}(\lambda)$ on a sheet of $T$ is bounded, which proves the theorem.

Remark 5.4. If the hypotheses of Theorem 5.3 are not satisfied, it may happen that $(\tilde{H}-\lambda)^{-1}$ has an essential singularity at $\lambda_{0} \in \Sigma$. This is due to the fact that the singular part of $(H-\lambda)^{-1}$ at $\lambda_{0}$ is in general not of finite rank.

\section{Appendix}

We prove here the result used in the proof of Corollary 4.12.

Proposition A.1. The residue $\tilde{\pi}_{0}$ of $\tilde{D}\left(p_{0}, \lambda, z_{0}\right) / f\left(p_{0}, \lambda, z_{0}\right)$ at $\lambda=\lambda_{0}$ satisfies the following identities:

$$
\left(H_{p_{0}}-\lambda_{0}+\tilde{R}_{0}\left(\lambda_{0}\right)\right) \tilde{\pi}_{0}=\tilde{\pi}_{0}\left(H_{p_{0}}-\lambda_{0}+\tilde{R}_{0}\left(\lambda_{0}\right)\right)=0 .
$$

Proof. Since $V \in L_{\alpha}^{k}\left(C_{T}\right), k>m, m \geqq 3$, using Sobolev inequalities, we see that $H_{p_{0}}$ 
is bounded between $H_{-a}^{1}\left(C_{T}\right)$ and $H_{-a}^{-1}\left(C_{T}\right)$, and between $H_{a}^{2}\left(C_{T}\right)$ and $L_{a}^{2}\left(C_{T}\right)$.

Let us put $\widetilde{G}\left(p_{0}, \lambda, z_{0}\right)=\widetilde{D}\left(p_{0}, \lambda, z_{0}\right) / f\left(p_{0}, \lambda, z_{0}\right)$. It is easy to see by analytic continuation that one has:

$$
\left(H_{p_{0}}-\lambda+\widetilde{R}_{0}(\lambda)\right) \tilde{G}\left(p_{0}, \lambda, z_{0}\right) \equiv \mathbf{1}_{1}, \widetilde{G}\left(p_{0}, \lambda, z_{0}\right)\left(H_{p_{0}}-\lambda+\widetilde{R}_{0}(\lambda)\right)=\mathbf{1}_{2},
$$

where $\mathbf{1}_{1}$ is the injection of $L_{a}^{2}\left(C_{T}\right)$ into $H_{-a}^{-1}\left(C_{T}\right)$ and $\mathbf{1}_{2}$ is the injection of $H_{a}^{2}\left(C_{T}\right)$ into $H_{-a}^{1}\left(C_{T}\right)$.

Then we have, if $\gamma$ is a small circle around $\lambda_{0}$ :

$$
\begin{aligned}
\left(H_{p_{0}}-\lambda_{0}+\tilde{R}_{0}\left(\lambda_{0}\right)\right) \tilde{\pi}_{0} & =\frac{1}{2 i \pi} \int_{\gamma}\left(H_{p_{0}}-\lambda_{0}+\tilde{R}_{0}\left(\lambda_{0}\right)\right) \tilde{G}\left(p_{0}, \lambda, z_{0}\right) d \lambda \\
& =\frac{1}{2 i \pi} \int_{\gamma} 1+O\left(\lambda-\lambda_{0}\right) \tilde{G}\left(p_{0}, \lambda, z_{0}\right) d \lambda=0 .
\end{aligned}
$$

On the other hand, let $\varphi \in H_{a}^{2}\left(C_{T}\right)$, and $u=\left(H_{p_{0}}-\lambda_{0}+\widetilde{R}_{0}\left(\lambda_{0}\right)\right) \varphi \in L_{a}^{2}\left(C_{T}\right)$. We have: $\widetilde{G}\left(p_{0}, \lambda, z_{0}\right) u=\varphi+\widetilde{G}\left(p_{0}, \lambda, z_{0}\right) O\left(\lambda-\lambda_{0}\right) \varphi$, so $\widetilde{G}\left(p_{0}, \lambda, z_{0}\right) u$ is holomorphic near $\lambda=\lambda_{0}$, which implies that $\tilde{\pi}_{0} u=0$. This proves the proposition.

Acknowledgements. We want to thank L. Guillopé for helpful discussions concerning Sect. III and J. Sjöstrand for pointing out an error in a first version of this work. We would also like thank

C. Sabbah for his generous help in complex analytic geometry.

\section{References}

[A] Alber, H. D.: A quasiperiodic boundary value problem for the Laplacian and the continuation of its resolvent. Proc. Roy. Soc. Edinburgh, 82A, 251-272 (1979)

[B.C] Balslev, E., Combes, J. M.: Spectral theory of many-body Schrödinger operators with dilation analytic interactions. Commun. Math. Phys. 22, 280-294 (1971)

[B.P] Bros, J., Pesenti, D.: Fredholm resolvents of meromorphic kernels with complex parameters: A Landau singularity and the associated equations of type $\mathscr{U}$ in a non-holomorphic case. J. Math. Pures Appl. 62, 215-252 (1983)

[C.F.K.S.] Cycon, H. L., Froese, R. G., Kirsch, W., Simon, B.: Schrödinger operators. Texts and Monographs in Physics. Berlin, Heidelberg, New York: Springer 1987

[F.K] Fukuda, T., Kobayashi, T.: A local isotopy lemma. Tokyo J. Math. 5, 31-36 (1982)

[F.H] Froese, R. G., Hislop, P.: Spectral analysis of second order elliptic operators on non-compact manifolds. Duke Math. J. 58, 103-128 (1989)

[Ge] Gerber, R. G.: Molecular scattering from surfaces: Theoretical methods and results. Chem. Review 29-79 (1987)

[G] Guillopé, L.: Théorie spectrale de quelques variétés à bouts. Ann. E.N.S. t22, 137-160 (1989)

[F.F.L.P] Fotiadi, D., Froissart, M., Lascoux, J., Pham, F.: Applications of an isotopy theorem. Topology 4, 159-191 (1965)

[Hi1] Hironaka, H.: Stratification and flatness. Proceedings of the Nordic Summer School. Oslo (1976)

[Hi2] Hironaka, H.: Bimeromorphic smoothing of complex analytic space. Acta Math. Vietnamica. Tome 2 (1977)

[H] Hislop, P.: Spectral analysis of non-compact manifolds using commutator methods. Séminaire E.D.P. Ecole Polytechnique (1988)

[K] Kobayashi, T.: On the singularities of the solution to the Cauchy problem with singular data in the complex domain. Math. Ann. 269, 217-234 (1984)

[L] Leray, J.: Le calcul différentiel et intégral sur une variété analytique complexe. Bull. S.M.F. 87, 81-180 (1959) 
[Me] Mercier, D. J.: Théorémes de régularité de type Nilsson. Thése de doctorat de l'Université de Nice (1984)

[Mi] Milnor, J.: Singular points of complex hypersurfaces. Princeton, NJ: Princeton Univ. Press 1968

[M] Mourre, E.: Absence of singular continuous spectrum for certain self-adjoint operators. Commun. Math. Phys. 78, 391-408 (1981)

[Mo] Moiseyev, N.: Complex scaling applied to trapping of atoms and molecules on solid surfaces. In: Lecture Notes in Physics, vol. 325. Berlin, Heidelberg, New York: Springer 1989

[P] Pham, F.: Introduction à l'étude topologique des singularités de Landau. Mémorial des sciences mathématiques ${ }^{\circ} 164$. Paris: Gauthier-Villars 1967

[Re-Si] Reed, M., Simon, B.: Methods of modern mathematical physics IV. Analysis of operators. London: Academic Press 1978

[Si] Simon, B.: Trace ideals and their applications. London Math. Soc. Lect. Notes vol. 35, Cambridge: Cambridge Univ. Press (1979)

[Sk] Skriganov, M. M.: Geometric and arithmetic methods in the spectral theory of multidimensional periodic operators. Proceedings of the Stekloc Institute of Mathematics $n^{\circ} 2$ (1987)

[V] Vaillant, J.: Ramification d'intégrales holomorphes. J. Math. pures et appl. 65, 343-402 (1986)

[Va] Vainberg, B. R.: On the analytic properties of the resolvent of a certain class of operator pencils. Math. Sb. 77, 199 (1968)

[W] Wilcox, C.: Scattering theory for diffraction gratings. Applied Mathematical Sciences $n^{\circ} 4.6$. Berlin, Heidelberg, New York: Springer 1980

Communicated by B. Simon

Received January 19, 1989; in revised form May 9, 1989 